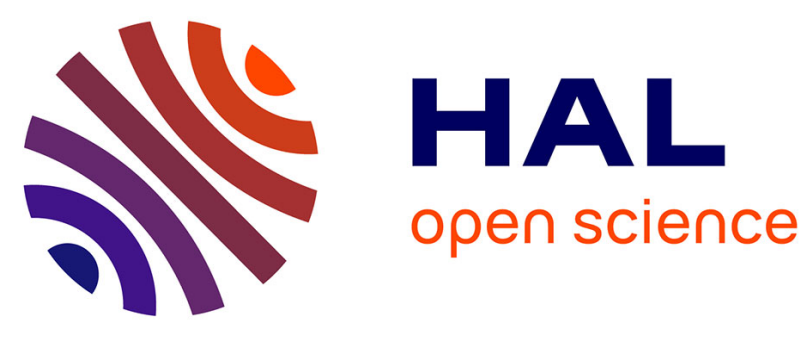

\title{
Comparison of Activity Monitors Accuracy in Assessing Intermittent Outdoor Walking
}

\author{
Aline Taoum, Ségolène Chaudru, Pierre-Yves de Müllenheim, Florian \\ Congnard, Mathieu Emily, Bénédicte Noury-Desvaux, Sandrine Bickert, Guy \\ Carrault, Guillaume Mahé, Alexis Le Faucheur
}

\section{To cite this version:}

Aline Taoum, Ségolène Chaudru, Pierre-Yves de Müllenheim, Florian Congnard, Mathieu Emily, et al.. Comparison of Activity Monitors Accuracy in Assessing Intermittent Outdoor Walking. Medicine and Science in Sports and Exercise, 2021, 53 (6), pp.1303-1314. 10.1249/MSS.0000000000002587. hal-03190738

\section{HAL Id: hal-03190738 \\ https://hal.science/hal-03190738}

Submitted on 23 Apr 2021

HAL is a multi-disciplinary open access archive for the deposit and dissemination of scientific research documents, whether they are published or not. The documents may come from teaching and research institutions in France or abroad, or from public or private research centers.
L'archive ouverte pluridisciplinaire HAL, est destinée au dépôt et à la diffusion de documents scientifiques de niveau recherche, publiés ou non, émanant des établissements d'enseignement et de recherche français ou étrangers, des laboratoires publics ou privés. 


\title{
Comparison of Activity Monitors Accuracy in Assessing Intermittent Outdoor Walking
}

\author{
Aline Taoum ${ }^{1}$, Ségolène Chaudru ${ }^{2}$, Pierre-Yves de Müllenheim ${ }^{3}$, Florian Congnard ${ }^{3}$, \\ Mathieu Emily ${ }^{4}$, Bénédicte Noury-Desvaux ${ }^{3}$, Sandrine Bickert ${ }^{5}$, Guy Carrault ${ }^{6}$, \\ Guillaume Mahé $^{2,7}$, Alexis Le Faucheur ${ }^{1,2}$
}

${ }^{1}$ University of Rennes 2, M2S - EA 7470, Rennes, France

${ }^{2}$ Clinical Investigation Center, INSERM 1414, University of Rennes 1, Rennes, France

${ }^{3}$ Institute of Physical Education and Sport Sciences (IFEPSA), UCO, Les Ponts-de-Cé, France

${ }^{4}$ Institut Agro, CNRS, Univ Rennes, IRMAR -UMR 6625, Rennes, France

${ }^{5}$ Laboratory of Vascular Investigations and Sports Medicine, University Hospital, Angers,

France

${ }^{6}$ Univ Rennes, Inserm, LTSI - UMR 1099, Rennes, France

${ }^{7}$ Heart-Vessels Imaging Team, University Hospital, Rennes, France

\section{CORRESPONDING AUTHOR DETAILS}

\section{Alexis LE FAUCHEUR}

Department of Sport sciences and physical education.

Ecole normale supérieure de Rennes

Campus de Ker Lann. Bruz, F-35170, France.

Tel: +33 (0)2 9905 9419. Fax: +33 (0)2 99059329 E-mail: alexis.lefaucheur@ens-rennes.fr 
Funding. The "Acti-GPS" project was promoted by the University Hospital of Angers and was partially funded by the Genesia Foundation. The "CLASH" project was promoted and partially funded by the University Hospital of Rennes (CORECT 2013). Both the "Acti-GPS" and "CLASH" projects were also partially funded by ENS Rennes. A. Taoum received a postdoctoral scholarship from the ENS Rennes and the Bretagne region (SAD funding). S. Chaudru received a PhD scholarship from the Bretagne region (ARED funding). P.-Y. de Müllenheim received a $\mathrm{PhD}$ scholarship from the ENS Cachan-Antenne de Bretagne (CDSN).

Conflicts of Interest. None. The results of the present study do not constitute endorsement by ACSM. The results of the study are presented clearly, honestly, and without fabrication, falsification, or inappropriate data manipulation. 


\begin{abstract}
Purpose: To determine and compare the accuracy of different activity monitors in assessing intermittent outdoor walking in both healthy and clinical populations through the development and validation of processing methodologies.
\end{abstract}

Methods: In study 1, an automated algorithm was implemented and tested for the detection of short ( $\leq 1 \mathrm{~min})$ walking and stopping bouts during prescribed walking protocols performed by healthy subjects in environments with low and high levels of obstruction. The following parameters obtained from activity monitors were tested, with different recording

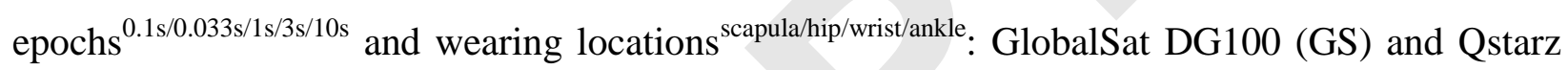
BT-Q1000XT/-Q1000eX (QS) speed; ActiGraph wGT3X+ (AG) vector magnitude (VM) raw data, VM counts, and steps; and StepWatch3 (SW) steps. Further, linear mixed models were developed to estimate walking speeds and distances from the monitors parameters. Study 2 validated the performance of the activity monitors and processing methodologies in a clinical population showing profile of intermittent walking due to functional limitations during outdoor walking sessions.

Results: In study $1, \mathrm{GS}^{1 \mathrm{~s} \text {, scapula }}, \mathrm{QS}^{1 \mathrm{~s} \text {, scapula/wrist }}$ speed, and $\mathrm{AG}^{0.033 \mathrm{~s} \text {, hip }} \mathrm{VM}$ raw data provided the highest bout detection rates $(>96.7 \%)$ and the lowest root-mean-square errors (RMSEs) in speed

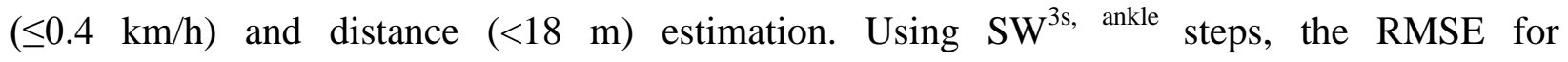
walking/stopping duration estimation reached $13.6 \mathrm{~min}$ using proprietary software and $0.98 \mathrm{~min}$

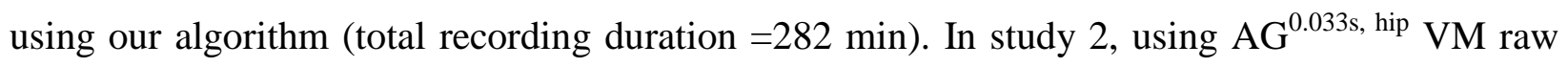


data, the bout detection rate [95\% CI] reached 100\% [99-100], and the mean absolute percentage errors (SD) in speed and distance estimation were 9\% (6.6) and 12.5\% (7.9), respectively.

Conclusion: GPS receivers and AG demonstrated high performance in assessing intermittent outdoor walking in both healthy and clinical populations.

KEY WORDS: Wearable monitors/devices, accelerometer, global positioning system, pedometer, physical activity, intermittent claudication. 


\section{INTRODUCTION}

Walking is recognized as the most popular, convenient, and free form of everyday physical activity (PA) and is thus of particular interest for improving health outcomes (1). Assessing and promoting outdoor walking is of importance since performing walking bouts at a sufficient intensity and of sufficient duration to meet PA recommendations may be easier outdoors and more enjoyable for people $(2,3)$. In addition to continuous walking, the total volume of ambulatory activity from day to day, regardless of the duration of the bouts of activity, is also of high importance to achieve health benefits $(4,5)$. Day-to-day human walking behaviors are intermittent by nature, as $76 \%$ and $69 \%$ of the total walking and stopping bouts, respectively, last less than 1 min (6). Furthermore, outdoor walking sessions are of primary interest in frail and clinical populations for both walking capacity assessment and rehabilitation purposes (7-9) since access to a supervised exercise program is limited in some clinical populations (10). The walking activity of people with functional limitations is also intermittent, including walking bouts of different durations and intensities, depending on their walking ability (11). The same is true regarding their ambulatory activity from day to day $(12,13)$. Therefore, regardless of the application, accurate monitors are needed to assess intermittent outdoor walking.

A full and accurate assessment of outdoor walking relies on a two-step approach: i) the identification of intermittent walking and stopping bouts that may be very short in duration and ii) the estimation of parameters related to intensity (e.g., walking speed or cadence) and volume (e.g., walking duration or distance or total steps) over identified walking bouts. There have been extensive studies throughout the years conducted on the use of pedometers, accelerometers, and global positioning system (GPS) monitors to assess walking (14-17). However, it is worth noting 
that a gap exists in the available studies regarding the accuracy of pedometers and accelerometers in providing a full assessment of short intermittent (outdoor) walking.

While extensive literature exists regarding the step count accuracy of pedometers during treadmill or natural walking (17-20), their accuracy in detecting walking/stopping bouts of different durations during outdoor intermittent walking is largely unknown. Moreover, the validity of using walking cadence from pedometers to estimate the speed of ambulation has not been investigated, but there is increasing interest in the assessment of such a parameter as a proxy indicator of the associated metabolic equivalent of task (MET) levels (21).

Accelerometers are the most used activity monitors in PA research but have been mainly used to assess PA patterns (not specifically walking) throughout periods $\geq 1 \mathrm{~min}$ in adults (22). Using machine learning algorithms, studies have classified activities under free-living conditions in adults on the basis of an accelerometer (at the hip or wrist level) and reported accuracies in walking (or stepping) identification ranging from $75 \%$ to $83 \%$ using sequential analysis windows of $10 \mathrm{~s}$ to $60 \mathrm{~s}$ of continuous activity (23-25). Although this approach is relevant, such algorithms are sophisticated and require a high level of technical skills, and the classification accuracy remains highly dependent on the initial database used for learning in terms of both the subject and activity characteristics.

Finally, GPS monitors, with or without accelerometers, have been used to study outdoor walking $(15,26)$, but only a few studies have focused on short intermittent walking $(14,27)$. Furthermore, the effects of the environment obstruction level and the wearing location on the accuracy of GPS 
monitors for such purposes are unknown.

The aim of the present study was to determine and compare the accuracy of different types of activity monitors in assessing intermittent outdoor walking. We focused on outdoor walking sessions considering the importance of implementing and thus monitoring home- or communitybased walking programs for public health $(7,9)$. For this purpose, we first developed and tested algorithms and prediction equations to assess the accuracy of GPS monitors, accelerometers, and pedometers in the estimation of intermittent outdoor walking during standardized and prescribed outdoor walking protocols in healthy participants (study 1). Herein, the analysis of walking activity relied on the detection of walking and stopping bouts and then quantifying walking bouts in terms of duration, speed, and distance. We aimed to implement an automated algorithm for bout detection that does not rely on the wearing location, recording epoch, or type of monitor used. Second, to study a real-world clinical application $(7,8)$, we validated the accuracy of the activity monitors and the developed processing methodologies during outdoor walking sessions performed by a group of people walking intermittently due to functional limitations (study 2).

\section{METHODS}

\section{Study overview}

The data and results of the present work were obtained from two distinct studies. Each study was approved by a local institutional ethics committee, and in both studies, the participants provided informed consent after being informed of the experimental procedure. Study 1 ("Acti-GPS" project; local institutional ethics committee: CPP OUEST II, Angers, France; NCT01805219) was specifically designed to include strict experimental conditions and be performed in healthy 
subjects to determine and compare the accuracy of different activity monitors in i) detecting intermittent outdoor walking bouts, for which algorithms were developed and tested, and ii) estimating walking speed and distance, for which prediction equations were developed and tested. Study 2 ("CLASH" project; local institutional ethics committee: CPP OUEST V, Rennes, France; NCT02041169) was a real-world scenario in which we validated the developed processing methodologies in a group of participants who experienced walking limitations during an outdoor walking session due to peripheral artery disease (PAD). More specifically, for these participants, their walking activity is limited due to lower-limb symptoms that impair their walking ability and force them to stop to recover.

\section{Study 1: Algorithm and equation development and testing}

Participants. Twenty healthy subjects $\left(23 \pm 3\right.$ years, $\left.71 \pm 10 \mathrm{~kg}, 176 \pm 8 \mathrm{~cm}, 22.7 \pm 3.0 \mathrm{~kg} / \mathrm{m}^{2}\right)$ were recruited. The inclusion criteria were as follows: being older than 18 years old; being affiliated with the social security system (according to French legislation); not presenting contraindications to the practice of physical activities; not being pregnant (women); and having read, understood, and signed the consent form.

Experimental procedure. For each participant, the experimental procedure consisted of an outdoor prescribed walking protocol (PWP). Ten participants (24 \pm 4 years, $71 \pm 9 \mathrm{~kg}, 177 \pm 8$ $\mathrm{cm}, 22.5 \pm 2.0 \mathrm{~kg} / \mathrm{m}^{2}$ ) each performed a different PWP on an outdoor flat athletic track (Rennes, France, latitude $=48.048184$, longitude $=-1.737166$ ) characterized by a low level of obstruction (LLO). The same PWPs were performed by the ten other participants ( $23 \pm 3$ years, $70 \pm 12 \mathrm{~kg}$, $176 \pm 9 \mathrm{~cm}, 22.9 \pm 3 \mathrm{~kg} / \mathrm{m}^{2}$ ) in a flat urban canyon (Angers, France, 47.468727, -0.541211) 
characterized by a high level of obstruction (HLO). Although there were 20 participants, we used only 10 different PWPs to compare the two environments, and each PWP was randomly assigned to one participant in each environment (LLO and HLO).

Each PWP was divided into two phases that were completed consecutively during the same session (Figure 1). The first phase of the PWP was used to develop (or improve) and test the accuracy of different algorithms in detecting intermittent walking and stopping bouts. This first phase was duration-based and relied on a stop-and-go pattern (14,27) during which the participants performed a sequence of walking and stopping bouts of fixed durations that were randomly selected from $\{3,6,12,15,20,30,40,50\}$ s (see document, Supplemental Digital Content 1, which details the design of the first phase of the PWP, http://links.lww.com/MSS/C238). The random sequence lasted between 10 and 15 min and was repeated twice by each participant: once at a "self-selected" walking pace and once again at a "slow" walking pace (Figure 1). The participants were equipped with an MP3 player that included instructions needed to alternate between bouts of walking and stopping, such as "start", “you will be stopping soon", "stop”, and "you will finish soon”.

The second phase of the PWP was used to develop and test the accuracy of the prediction equations for speeds and distances over the detected walking bouts. This phase was distancebased, and each participant repeated a sequence of ten walking bouts of fixed distances separated by stopping bouts of $\sim 30 \mathrm{~s}$. There were five predetermined walking distances of $25,50,75,100$, and $200 \mathrm{~m}$, which were each repeated twice and randomly distributed within the sequence. The sequence was repeated by each participant at a "self-selected", "slow" and then "fast" pace 
(Figure 1). In this second phase, cones were placed every $25 \mathrm{~m}$ on the outdoor athletic track or the urban canyon. The actual distances were measured using an odometer. The participants were asked to walk and stop as instructed by the investigator who supervised the PWP. In the urban canyon, when the participants arrived at the cone at either end of the pathway, they were asked to turn around without stopping and continue walking in the opposite direction.

All the PWPs were supervised by an investigator who followed the protocol sequence using a stopwatch and recorded the time elapsed during each bout.

Instrumentation. A total of 11 research-grade activity monitors were worn simultaneously by each participant during the PWPs, including seven GPS monitors, two accelerometers, and two pedometers. More specifically, one GlobalSat DG100 GPS receiver (GS, GlobalSat Technology Corp., Taiwan) that recorded data for 1-s epochs was worn in a shoulder pack with an external antenna fixed on the top of the shoulder strap at the level of the right scapula $\left(\mathrm{GS}^{1 \mathrm{~s}, \text { scapula }}\right)$. Three Qstarz BT-Q1000XT and three Qstarz BT-Q1000eX GPS receivers (QS, Qstarz International Co., Ltd., Taipei, Taiwan) that recorded data for 1-s and 0.1-s (10Hz) epochs, respectively, were

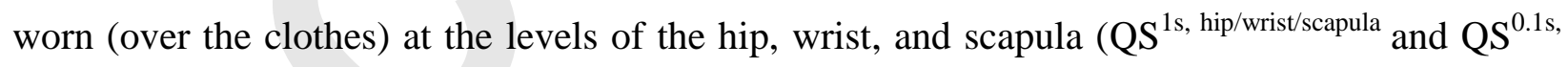
hip/wrist/scapula $)$. The GPS receivers were initialized before all the experiments to ensure there was good reception of the satellites' signals. Two wGT3X+ accelerometers (AG, ActiGraph ${ }^{\mathrm{TM}}, \mathrm{LLC}$ firmware version: 2.2.1-2.4.0, Shalimar, FL, USA) were programmed for recording raw data at a recording rate of $30 \mathrm{~Hz}(0.033 \mathrm{~s})$ and were worn at the levels of the hip (right or left) and wrist

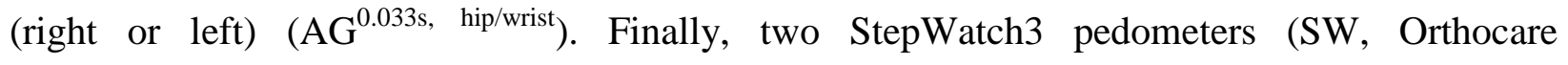
Innovations, Washington, D.C.) were worn on each ankle; they recorded data for 3-s and 10-s 
epochs each $\left(\mathrm{SW}^{3 \mathrm{~s} / 10 \mathrm{~s}, \text { ankle }}\right)$ and were set to the default settings. SW monitors were available only for the experiments performed in the LLO environment. For each category of activity monitors, the wearing location was randomly selected.

Data extraction and preprocessing. After each experiment, the data were downloaded to a personal computer using the appropriate manufacturer's software for each activity monitor. The GS and QS data were downloaded using the Data Logger PC Utility (version 1.1, GlobalSat Technology Corp., Taiwan) and QTravel software (version 1.49, Qstarz International Co., Ltd., Taipei, Taiwan), respectively, and were automatically expressed in speed $(\mathrm{km} / \mathrm{h})$. The AG data were extracted using ActiLife 6 software (versions $\geq 6.5 .3$, ActiGraph ${ }^{\mathrm{TM}}$, LLC Shalimar, FL, USA) in three forms, namely, the raw acceleration (G-Force) in $0.033 \mathrm{~s}$ epochs and the counts and the steps in $1 \mathrm{~s}$ epochs. The counts, computed from the raw acceleration, and the steps were obtained using two different methods: the normal filter (NF) and the low frequency extension filter (LFE). The raw acceleration and counts were then used to calculate the corresponding vector magnitude $(\mathrm{VM})$, defined by $\sqrt{x^{2}+y^{2}+z^{2}}$, where $x, y$, and $z$ represent the raw acceleration or the counts provided from each axis. In this study, we resampled the $\mathrm{AG}^{0.033 s \text {, }}$ hip/wrist $\mathrm{VM}$ raw acceleration and the $\mathrm{QS}^{0.1 \mathrm{~s} \text {, hip/wrist/scapula }}$ speed to $1 \mathrm{~s}$ to remove unnecessary and noisy information. Herein, for the sake of simplicity, "AG $\mathrm{AG}^{0.033 \mathrm{~s},{ }^{*}} \mathrm{VM}$ raw data" and " $\mathrm{AG} \mathrm{G}^{1 \mathrm{~s},{ }^{*}} \mathrm{VM}$ counts" refer to the VM computed from the resampled raw acceleration and the counts/s for a

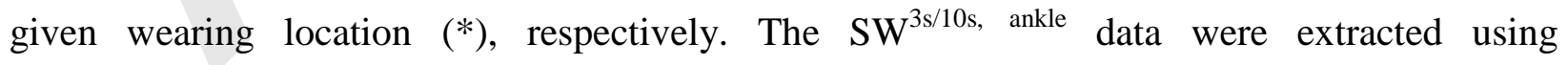
StepWatch $^{\mathrm{TM}}$ software (version 3.2, Orthocare Innovations, Washington, D.C.) in files including $\mathrm{SW}^{3 \mathrm{~s} / 10 \mathrm{~s} \text {, ankle }}$ steps and a timestamp for the corresponding recording epoch. The activity duration (i.e., walking duration) and nonactivity duration (i.e., stopping period) calculated by the SW 
software for each minute were also reported. To facilitate further data analysis, the activity/nonactivity duration algorithm used by the SW software was implemented in MATLAB® 2018b (MathWorks, Massachusetts, USA).

Algorithm and equation development. As previously mentioned, we aimed to assess intermittent outdoor walking by first identifying intermittent walking from stopping bouts and second, by estimating the walking speed and distance over the walking bouts that were detected.

Hence, the first objective was to develop a new algorithm for the detection of walking and stopping bouts offline that can assess each of the following parameters obtained from the

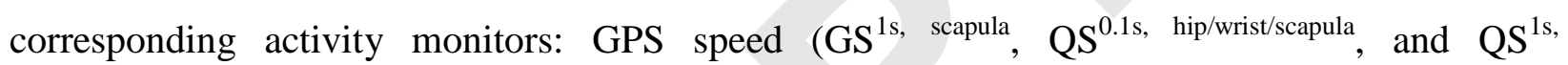

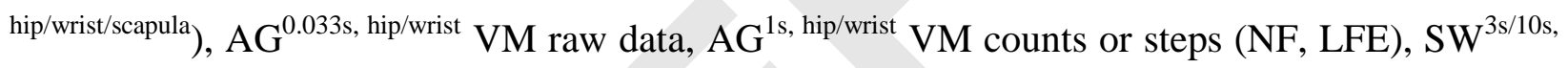
ankle steps. To develop and test the algorithm, only the first phase (duration-based phase) of the performed PWPs was considered. From the time series of a given parameter obtained for a given participant, the algorithm determined the best threshold that divided the time series between walking and stopping by constructing a histogram of the considered time series. Then, the threshold was determined as the first local minima of the generated distribution. Any value below this threshold was identified as a stopping event, and all other values were identified as walking events. We then gathered all consecutive events of stopping and walking in bouts of stopping and walking, respectively. Then, for further analysis, bouts were then characterized by their status (walking/stopping), their time of occurrence, and their durations. The proposed method is commonly used in image processing applications and is known as the watershed algorithm (28). Figure 2 illustrates an example of the proposed algorithm applied to GPS speed. 
In the same context, we were also interested in enhancing our previously validated algorithm (14), which was specifically designed for GPS speed only. Hence, we proposed to improve and automate the original algorithm (see document, Supplemental Digital Content 2, which describes the previous algorithm and its optimization, http://links.lww.com/MSS/C239). We also tested the enhanced algorithm on each studied parameter obtained from the activity monitors in addition to the GPS receivers. The algorithms were developed and implemented in MATLAB® $2018 \mathrm{~b}$.

The second objective was to develop and test the accuracy of prediction equations in estimating the speeds and distances of each detected walking bout. To address this second objective, only the second phase (distance-based phase) of the PWPs was considered. The walking and stopping bouts were first detected using the developed watershed algorithm. Then, for the GPS receivers, the mean GPS speed and the GPS distance were calculated for each detected walking bout. The GPS distance was computed from the mean GPS speed and the corresponding bout duration. For

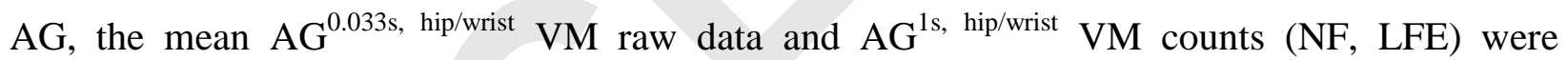
computed for each detected walking bout. The total VM was also computed from the VM raw data and VM counts (NF, LFE) by summing the VM values for each walking bout. For $\mathrm{SW}^{3 s / 10 s \text {, }}$ ankle steps and $\mathrm{AG}^{1 \mathrm{~s}, \text { hip/wrist }}$ steps (NF, LFE), the step cadence was computed for each walking bout from the sum of the recorded step counts and the corresponding bout duration and was then expressed in steps/min. The total number of steps was also computed for each walking bout from $\mathrm{AG}^{1 \mathrm{~s} \text {, hip/wrist }}$ steps $(\mathrm{NF}, \mathrm{LFE})$ and $\mathrm{SW}^{3 \mathrm{~s} / 10 \mathrm{~s} \text {, ankle }}$ steps. Thereafter, prediction equations were developed using linear mixed models (LMMs) to estimate the actual walking speed and distance from the corresponding parameters measured by each activity monitor for each walking bout: i) GPS mean speed, $\mathrm{AG}^{0.033 \mathrm{~s}, \text { hip/wrist }}$ mean VM raw data, $\mathrm{AG}^{1 \mathrm{~s} \text {, hip/wrist }}$ mean VM counts and step 
cadence (NF, LFE), and $\mathrm{SW}^{3 \mathrm{~s} / 10 \mathrm{~s} \text {, ankle }}$ step cadence for speed estimation; ii) GPS distance, $\mathrm{AG}^{0.033 \mathrm{~s}, \text { hip/wrist }}$ total $\mathrm{VM}$ raw data, $\mathrm{AG}^{1 \mathrm{~s} \text {, hip/wrist }}$ total VM counts and total steps (NF, LFE), and $\mathrm{SW}^{3 \mathrm{~s} / 10 \mathrm{~s} \text {, ankle }}$ total steps for distance estimation. The average actual speed during each walking bout was computed by dividing the actual distance of each bout by the elapsed time, as measured by the investigator using the stopwatch. The equations obtained for speed estimation from a given activity monitor were also used to indirectly estimate walking distance. For the GPSs, the equations were developed for each environment separately, as well as for the combined environments. For the AG, the equations were developed for the combined environments only. The LMM approach was used to account for the dependence among repeated measurements obtained from the same subject (29). Let $\hat{Y}_{S}(j)$ be the speed/distance estimation for the $j^{\text {th }}$ walking bout of subject $s$. This estimation can be modeled from the studied parameter $p_{s}(j)$ using the LMM as follows:

$$
\hat{Y}_{s}(j)=\alpha+\beta \times p_{s}(j)+a_{s}+b_{s} \times p_{s}(j)+\varepsilon
$$

where $\alpha$ and $\beta$ are fixed effects coefficients related to the population's characteristics, $a_{s}$ and $b_{s}$ denote the individual subject's random intercept and slope deviation, respectively, and $\varepsilon$ represents the random error. The models were fitted using the restricted maximum likelihood method (30). A leave-one-out cross-validation (LOOCV) approach was used to build and evaluate the LMMs. The modeling was performed using R software 2020 (R Foundation for Statistical Computing, Vienna, Austria, version R.4.0.0).

Statistical analysis. First, for each monitor, the performance of the algorithms in detecting 
walking and stopping bouts was analyzed at the "bout-level" $(14,27)$ using the detection rate, which was defined by the percentage of correctly detected bouts and was computed as follows:

$$
\text { detection rate }(\%)=\frac{\text { Number of correctly detected walking and stopping bouts }}{\text { Total number of bouts }} \times 100 \text {. }
$$

A bout was considered correctly detected if it corresponded to the actual bout of the PWP and if its duration was within $\pm 20 \%$ of the actual bout duration (27). The detection rates were expressed with their $95 \%$ confidence intervals (95\% CIs).

Furthermore, we used the McNemar or the Cochran Q statistical test to compare the bout detection rates across the various conditions studied, such as the monitor wearing locations, environments, and types of monitors. The McNemar test is a nonparametric statistical test appropriate for nominal dichotomous data and was used to compare the proportions of identified/misidentified bouts between two studied conditions. When more than two studied conditions were compared, the Cochran Q test was used (31), and the Bonferroni correction method for multiple comparisons was used. P values $<0.05$ were considered statistically significant. Statistical analysis for bout detection was performed in MATLAB® 2018b.

Second, the performance of the LMM-based prediction equations was evaluated using $\mathrm{R}$ software 2020 by computing $\mathrm{R}^{2}$, the standard error of estimate (SEE), and Akaike information criteria (AIC) value. The AIC value reflects both the goodness of fit and the efficiency of a model for a given number of predictor variables. The lower the AIC value, the better the quality of the model is. For each model, the error in the estimation of speed and distance following the 
leave-one-out procedure was computed from the average root-mean-square error (RMSE).

\section{Study 2: Validation in a clinical population}

Participants. Twenty-three participants with PAD with exertional limb symptoms $(60 \pm 10$ years, $78.9 \pm 16.3 \mathrm{~kg}, 169 \pm 8 \mathrm{~cm}, 27.4 \pm 5.0 \mathrm{~kg} / \mathrm{m}^{2}$ ) were recruited from the Vascular Medicine Unit (University Hospital, Rennes, France) to participate in the "CLASH" project. All participants were diagnosed with PAD and were limited to walking during treadmill tests. For a detailed description of the inclusion and exclusion criteria of the "CLASH" protocol, please refer to (32).

Experimental procedure. During the "CLASH" protocol, the participants with PAD were asked to perform an outdoor walking session at their self-selected walking pace for $45-60 \mathrm{~min}$, including bouts of stopping (recovery) due to lower-limb pain. In the "CLASH" protocol, the stopping durations performed by each participant were determined on the basis of a random sequence of the following stop durations, $0.5,1.5,2.5,3.5,4.5 \mathrm{~min}$, and a self-selected duration determined by each participant (depending on symptom relief). The walking session was performed on the same outdoor athletic track (LLO) as for the healthy participants and supervised by an investigator who recorded the duration of each walking and stopping bout using a stopwatch.

Instrumentation and data extraction. Only a selection of the activity monitors tested in the first study was used in this clinical study. The participants with PAD wore one GS ${ }^{1 \text { s, scapula }}$, one $\mathrm{AG}^{0.033 \mathrm{~s}, \text { hip }}$, and one $\mathrm{SW}^{10 \mathrm{~s} \text {, ankle }}$. The GS and AG were configured as detailed in study 1 . For the 
SW, the "walking speed" and "leg motion" settings were set according to the gait characteristics of the participants, as recommended by the manufacturer. For all monitors, the data were downloaded and preprocessed following the same procedure as detailed in study 1 .

Data analysis. The watershed algorithm developed in study 1 was applied to the parameters collected from the participants in study 2 to test the performance of the algorithm in detecting walking and stopping bout in a clinical application. In addition, once the watershed algorithm was applied, we aimed to test the use of additional filters of $2 \mathrm{~s}, 10 \mathrm{~s}$ and $15 \mathrm{~s}$ to remove bouts $\leq 2$ $\mathrm{s},<10 \mathrm{~s}$, and $<15 \mathrm{~s}$. Such filters have been previously used $(8,11,14)-$ but never formally tested - in participants with PAD. The 2 s-filter was initially proposed as an artifact management tool to remove very short walking and stopping bouts (14). The $10 \mathrm{~s}$ - and $15 \mathrm{~s}$-filters were selected on the basis of the clinical rationale that walking-induced ischemia is unlikely to elicit maximal claudication pain (during walking) or to disappear (during stopping) for such short periods (11). It is worth noting that only the $15 \mathrm{~s}$ filter was applied on SW data due to its recording epoch. Thereafter, for each detected walking bout, the walking speed and distance were estimated from the LMM prediction equations developed in study 1.

Statistical analysis. First, the walking and stopping bout detection performance was computed as previously defined to compare all the detected bouts with the actual walking and recovery (stopping) bouts, (i.e., the detection rate was defined as the percentage of correctly detected walking and stopping bouts). The detection rates when using additional filters of $2 \mathrm{~s}, 10 \mathrm{~s}$, and 15 s were also computed. Second, to determine the performance of speed and distance estimation over each detected walking bout, the $\mathrm{GS}^{1 \mathrm{~s} \text {, scapula }}$ speeds and distances were considered as the 
reference (actual) values. Then, the following error metrics were computed: the bias of estimation, the typical error of estimate (TEE), the coefficient of variation (CV), the mean percent error (MPE), and the mean absolute percent error (MAPE) (33,34). TEE and CV are presented with their 95\% CI, whereas MPE and MAPE are presented with their standard deviation.

\section{RESULTS}

For better readability, only a selection of the most significant findings is presented below (see Worksheets, Supplemental Digital Content 3, which details all the results of the study, http://links.lww.com/MSS/C240).

\section{Study 1: Algorithm and equations development and testing}

The participants performed a total of 768 walking and 768 stopping bouts during the first phase of the PWP. QS ${ }^{1 \text { s, hip }}$ and $\mathrm{AG}^{0.033 \text { s, hip }}$ recordings were each missing for one participant in the LLO environment. The mean \pm standard deviation (range) of walking speed for GS ${ }^{1 \text { s, scapula }}$ was $4.6 \pm$ $0.7 \mathrm{~km} / \mathrm{h}(1.6-6.34 \mathrm{~km} / \mathrm{h})$ for the "self-selected" pace and $3.3 \pm 0.7 \mathrm{~km} / \mathrm{h}(1.1-5.3 \mathrm{~km} / \mathrm{h})$ for the "slow" pace. During the second phase of the PWP, the participants performed a total of 600 walking and 600 stopping bouts. Two episodes of GPS signal loss over two different walking bouts, lasting $14 \mathrm{~s}$ and $34 \mathrm{~s}$ each, were noted for the GS ${ }^{1 \mathrm{~s}}$ scapula receiver. Hence, these two walking bouts were excluded from the analysis. The mean \pm standard deviation (range) of GS ${ }^{1 s \text {, }}$ scapula walking speed was $4.9 \pm 0.6 \mathrm{~km} / \mathrm{h}(3.4-6.5 \mathrm{~km} / \mathrm{h})$ for the "self-selected" pace, $3.9 \pm 0.6$ $\mathrm{km} / \mathrm{h}(2.6-5.5 \mathrm{~km} / \mathrm{h})$ for the "slow" pace, and $5.9 \pm 0.7 \mathrm{~km} / \mathrm{h}(4.4-7.6 \mathrm{~km} / \mathrm{h})$ for the "fast" pace. 
Performance for bout detection. The results related to the detection rates of walking and stopping bouts are presented in Table 1 and Figure 3. Table 1 shows that overall, both algorithms (the watershed and the enhanced algorithms) provided close detection rates across almost all the studied parameters. When considering all the results obtained from the watershed algorithm, regardless of the level of obstruction due to the environment, the highest detection rates for GPS measurements corresponded to $\mathrm{GS}^{1 \mathrm{~s} \text {, scapula }}$ and $\mathrm{QS}^{1 \mathrm{~s} \text {, wrist/scapula }}$ speeds with detection rates of 96.6\% [95.5 - 97.4], 96.7\% [95.7 - 97.5], and 96.4\% [95.4 - 97.3], respectively. The detection rate of the $\mathrm{QS}^{1 \mathrm{~s} \text {, hip }}$ speed was $91.3 \%$ [89.7 - 92.7] and was significantly lower than those obtained from $\mathrm{QS}^{1 \mathrm{~s} \text {, wrist/scapula }}(\mathrm{P}<0.001)$. Moreover, $\mathrm{QS}^{0.1 \text { s, hip/wrist/scapula }}$ had the lowest detection rates across wearing locations among the GPS measurements $(\mathrm{P}<0.05)$. All $\mathrm{AG}^{0.033 s, \text { hip }}$ and $\mathrm{AG}^{1 \mathrm{~s} \text {, hip }}$ parameters (i.e., VM raw data, VM counts, and steps) had significantly better performance than did the $\mathrm{AG}^{0.033 \text { s, wrist }}$ and $\mathrm{AG}^{1 \mathrm{~s} \text {, wrist }}$ parameters $(\mathrm{P}<0.05)$, with a detection rate of $98.3 \%$ [97.5 - 98.9] for $\mathrm{AG}^{0.033 \mathrm{~s} \text {, hip }} \mathrm{VM}$ raw data. There was no significant difference between $\mathrm{AG}^{0.033 s, \text { hip }} \mathrm{VM}$ raw data and $\mathrm{AG}^{1 \mathrm{~s} \text {, hip }} \mathrm{VM}$ counts (NF, LFE). The detection rate of $\mathrm{SW}^{3 \mathrm{~s} \text {, ankle }}$ steps was $87.6 \%$ [85.1 - 89.9], which was significantly higher than that of $\mathrm{SW}^{10 \text { s, ankle }}$ steps, which was $21.8 \%$ [18.9 - 24.8]. Although the detection rates obtained from $\mathrm{SW}^{3 \mathrm{~s} / 10 \mathrm{~s} \text {, ankle }}$ steps were lower, the error in detecting the total actual duration of activity (141 min) and inactivity (141 min) was considerably lower when the watershed algorithm rather than the SW software was used (RMSE of activity/inactivity duration estimation: $0.98 \mathrm{~min}$ vs. $13.6 \mathrm{~min}$ for $\mathrm{SW}^{3 \mathrm{~s} \text {, ankle }}$ steps, and $3.5 \mathrm{~min}$ vs. $12.4 \mathrm{~min}$ for $\mathrm{SW}^{10 \mathrm{~s} \text {, ankle }}$ steps).

For inter-monitors comparisons, the detection rate was slightly but significantly higher for $\mathrm{AG}^{0.033 s, \text { hip }} \mathrm{VM}$ raw data and $\mathrm{AG}^{1 \mathrm{~s} \text {, hip }} \mathrm{VM}$ counts $(\mathrm{NF})$ than for $\mathrm{GS}^{1 \mathrm{~s} \text {, scapula }}$ speed and $\mathrm{QS}^{1 \mathrm{~s} \text {, }}$ 
wristscapula speed $(\mathrm{P}<0.05)$. Furthermore, $\mathrm{SW}^{3 s / 10 \text { s, ankle }}$ steps resulted in a significantly lower detection rate than did the GPS speeds $\left(\mathrm{GS}^{1 \mathrm{~s} \text {, scapula }}\right.$ and $\left.\mathrm{QS}^{0.1 \mathrm{~s} / 1 \mathrm{~s} \text {, hip/wrist/scapula }}\right), \mathrm{AG}^{0.033 s \text {, hip/wrist }} \mathrm{VM}$ raw data and $\mathrm{AG}^{1 \mathrm{~s}, \text { hip/wrist }} \mathrm{VM}$ counts $(\mathrm{P}<0.05)$.

Figure 3 shows the effects of both the duration of the bouts and the level of obstruction on the bout detection rate for a selection of monitors, wearing locations, and recording epochs. Regardless of the monitor, wearing location, and recording epoch, the detection rate was the lowest for short bouts and then increased for longer bouts (Figure 3 and see Worksheet Study1_Boutdetection, Supplemental Digital Content 3, which details all the results of the study, http://links.lww.com/MSS/C240). $\mathrm{QS}^{1 \mathrm{~s} \text {, wrist }}$ speed and $\mathrm{AG}^{0.033 s, \text { hip }} \mathrm{VM}$ raw data had similar detection rates $(98 \%$ [96 - 99] vs. 98\% [97 - 99], P >0.05) in LLO environment. The detection rate of $\mathrm{QS}^{1 \mathrm{~s} \text {, wrist }}$ speed decreased in HLO environment compared with LLO (96\% [94 - 97] vs 98\% [96 - 99], P <0.05) and became lower (96\% [94 - 97] vs. 98\% [97 - 99], P <0.05) than that of $\mathrm{AG}^{0.033 \text { s, hip }} \mathrm{VM}$ raw data (Figure 3).

Estimation of speed and distance. Table 2 presents a selection of the results for all monitors from LMM-based speed prediction equations and the LOOCV procedure. Estimation models with a higher $\mathrm{R}^{2}$ and lower RMSE were obtained from GPS speeds in the LLO environment (see Worksheet Study1_LMMEquations, Supplemental Digital Content 3, which details all the results of the study. http://links.lww.com/MSS/C240). When the results obtained from both environments were considered, the model using $\mathrm{AG}^{0.033 s, \text { hip }} \mathrm{VM}$ raw data yielded a similar RMSE as those obtained from the GPS speeds. For the estimation of walking distance, the models using GPS distance demonstrated better prediction performance than did the models 
developed using $\mathrm{AG}^{0.033 \mathrm{~s} \text {, hip/wrist }} \mathrm{VM}$ raw data, $\mathrm{AG}^{1 \mathrm{~s} \text {, hip/wrist }} \mathrm{VM}$ counts, steps, and $\mathrm{SW}^{3 \mathrm{~s} / 10 \mathrm{~s} \text {, ankle }}$ steps, as shown by the lower SEE and RMSE values, higher $\mathrm{R}^{2}$ coefficients and better AIC.

\section{Study 2: Validation in a clinical population}

A total of 149 walking and 127 stopping bouts were performed by the PAD participants during the outdoor walking sessions. No data loss was noted. The mean \pm SD (range) of GS ${ }^{1 \mathrm{~s} \text {, scapula }}$ walking speed was $4.2 \pm 0.5 \mathrm{~km} / \mathrm{h}(3.3-5.5 \mathrm{~km} / \mathrm{h})$. Table 3 presents the bout detection rate as well as the accuracy of speed and distance estimations when the $15 \mathrm{~s}$ filter was applied.

Bout detection. Walking and stopping bouts were detected with a detection rate of $100 \%$ when the $15 \mathrm{~s}$ filter was applied for $\mathrm{GS}^{1 \mathrm{~s} \text {, scapula }}$ speed, $\mathrm{AG}^{0.033 \mathrm{~s} \text {, hip }} \mathrm{VM}$ raw data, and $\mathrm{AG}^{1 \mathrm{~s} \text {, hip }} \mathrm{VM}$ counts (NF, LFE). Lower detection rates were obtained from $\mathrm{AG}^{1 \mathrm{~s} \text {, hip }}$ steps $(\mathrm{NF})$ and $\mathrm{SW}^{10 \text { s, ankle }}$ steps. When only the 2 s filter was applied (see Worksheets Study2_Boutdetection and Study2_Estimations, Supplemental Digital Content 3, which details all the results of the study, http://links.lww.com/MSS/C240), the detection rates of $\mathrm{AG}^{1 \mathrm{~s} \text {, hip }} \mathrm{VM}$ counts and steps (NF, LFE) decreased, while the detection rates for $\mathrm{GS}^{1 \mathrm{~s} \text {, scapula }}$ speed and $\mathrm{AG}^{0.033 \mathrm{~s} \text {, hip }} \mathrm{VM}$ raw data remained high at 98.6\% [96 - 99] and 99.6\% [98 - 100], respectively. Additionally, the error in detecting the total actual duration of activity (605 $\mathrm{min}$ ) and inactivity (309 $\mathrm{min})$ was lower when the watershed algorithm rather than the SW software was used (RMSE of activity/inactivity duration estimation: $0.81 \mathrm{~min}$ vs $10.1 \mathrm{~min})$.

Estimation of speed and distance. The MAPE obtained from the speed and distance estimations in the PAD participants are presented in Table 3 (see also Worksheet Study2_Estimations, 
Supplemental Digital Content 3, which details all the results of the study, http://links.lww.com/MSS/C240). The $\mathrm{AG}^{0.033 s, \text { hip }} \mathrm{VM}$ raw data presented the lowest MAPE in estimating the walking speed using the developed LMM equations. Consequently, it showed the best estimation of distance when both the LMM equations and the estimated speed were used.

\section{DISCUSSION}

The main findings of the present study can be summarized as follows:

a) Both of the algorithms tested were comparably accurate in intermittent walking bout detection.

b) High and comparable detection rates of outdoor walking and stopping bouts in the environment with LLO were obtained from the GPS speeds $\left(\mathrm{GS}^{1 \mathrm{~s} \text {, scapula }}\right.$ and $\mathrm{QS}^{0.1 \mathrm{~s} / 1 \mathrm{~s} \text {, hip/wrist/scapula }}$ ) and accelerometer parameters $\mathrm{AG}^{0.033 \mathrm{~s} \text {, hip }} \mathrm{VM}$ raw data and $\mathrm{AG}^{1 \text { s, hip }} \mathrm{VM}$ counts; however, the detection rate decreased in the HLO environment for GPS speeds and became significantly lower than those obtained from $\mathrm{AG}^{0.033 \text { s, hip }} \mathrm{VM}$ raw data and $\mathrm{AG}^{1 \mathrm{~s} \text {, hip }} \mathrm{VM}$ counts.

c) In the LLO environment, the GPS speeds $\left(\mathrm{GS}^{1 \mathrm{~s} \text {, scapula }}\right.$ and $\left.\mathrm{QS}^{0.1 \mathrm{~s} / 1 \mathrm{~s} \text {, hip/wrist/scapula }}\right)$ yielded better estimations of walking speed, whereas when both environments were considered, $\mathrm{AG}^{0.033 \text { s, hip }}$ VM raw data was more accurate and robust.

d) The wearing location had a large and significant effect on monitor performance in bout detection and in the estimation of speed and distance; the GPS had the best performance at the wrist and scapula levels, and the AG device had the best performance at the hip level. 
e) The $\mathrm{SW}^{3 \mathrm{~s} / 10 \mathrm{~s} \text {, ankle }}$ step parameter was less accurate in identifying walking bouts than were the GPS and AG monitors, although its accuracy was improved when the new proposed algorithm rather than the default SW software was used.

f) For the clinical application, the GPS and AG monitors provided the highest accuracy in assessing the intermittent walking activity of patients with functional limitations.

\section{Subject-based, automated algorithm for the detection of walking and stopping bouts.}

In contrast to all existing studies that focused on detecting longer walking sessions by applying standard thresholds for speed and/or accelerometer data, the new algorithm proposed in the present study is subject-based and is not affected by the type of monitor (parameter), wearing location, or recording epoch used. In previous studies, walking trips were defined as $3-5$ consecutive min of walking at speeds between 2 and $6-8 \mathrm{~km} / \mathrm{h}$ over distances $>30 \mathrm{~m}$ or accelerometer counts $>500$ counts/epoch $(15,26,35)$. These walking bouts were interspersed with stopping bouts of durations $>3$ min. However, these studies did not consider the fragmented nature of day-to-day walking behavior (6), which precludes the use of such methods for the precise assessment of intermittent outdoor walking, both in healthy and clinical populations. In this present study, our previous algorithm for bout detection was also improved and became fully automated (14). The watershed algorithm had generally comparable detection rates to those of the enhanced version of our previous algorithm, and it had lower computational complexity because it consisted of a one-step algorithm and did not require a learning phase or the optimization of parameters. Although the enhanced version of our previous algorithm presented 
high and promising detection rates, it was developed and tested on a limited dataset of healthy participants. A higher number of participants is required to carry out the learning procedure on different walking profiles and then to generalize the algorithm to the whole population. For the clinical application (study 2), $2 \mathrm{~s}, 10 \mathrm{~s}$ or $15 \mathrm{~s}$ filters were applied according to previous studies $(8,11,14)$ to remove short walking displacement data that reflected patients recovering from the pain symptoms induced by ischemia during the previous walking bout. These short walking displacements were real (and detected) but had no clinical significance in the present context. However, in another context, if one aims to estimate total ambulatory (outdoor) activity throughout one day, short bouts lasting $<10 \mathrm{~s}$ or $15 \mathrm{~s}$ may be of interest when for instance, one is investigating activities around the house such as gardening or other chores. Thus, the $10 \mathrm{~s}$ or $15 \mathrm{~s}$ filter should not be applied in these contexts. According to the context, the processing methodology needs to be adjusted when data from activity monitors are analyzed. Furthermore, despite the high detection rates of the GPS and accelerometer monitors, the watershed algorithm had a lower detection rate when the $\mathrm{SW}^{10 \mathrm{~s} \text {, ankle }}$ was used due to the shorter recording epoch. The criterion we used to determine whether a bout was correctly detected ( $\pm 20 \%$ of actual duration) may also affect the bout detection rate. For instance, for the $\mathrm{SW}^{10 \mathrm{~s} \text {, ankle }}$, when criterion values of $10 \%, 20 \%$, and $30 \%$ were tested, the bout detection rate varied accordingly to $90 \%, 95 \%$, and $97 \%$, respectively.

\section{Accuracy of GPS speed in assessing intermittent outdoor walking.}

GPS receivers have been primarily used to determine how the physical environment is associated with the PA behaviors of individuals $(26,36)$. The present study extends previous works that have used GPSs to assess outdoor walking activity bouts $\geq 1 \min (15,26,35,37)$. Our results also 
extend previous studies $(14,27)$ that addressed the validity of GPS receivers in assessing intermittent outdoor walking and show how performance is affected by the environment's obstruction level, the device wearing location, and recording epoch. The QS GPS monitor is a good alternative to the GS, which is considered accurate but no more marketed. QS is among the most commonly used GPS monitors by PA researchers for long-term real-life measurements $(26,37)$ and is generally placed at the hip level along with an accelerometer. According to our results, the QS monitor provides a lower bout detection rate $(\sim 91 \%)$ at the hip level than at the wrist and scapula levels, which are uncommon locations for long-term QS measurements. This finding should be considered by users in future studies. Furthermore, although it has been shown - mainly in sports studies - that high-frequency GPS monitors can enhance the accuracy of distance and speed estimation compared with low-frequency GPS monitors (38), our results showed that they are less accurate in identifying walking bouts since the signals have high variability and need additional processing steps for undersampling. They also cannot be used in real-life scenarios in which data needs to be recorded for several days. The error of the speed and distance estimations obtained in our study were consistent with those reported in previous studies $(27,39)$ for LLO conditions. However, we provided the first data regarding the effects of the level of obstruction and wearing location on the accuracy of estimating walking speed and distance.

GPS monitors have been previously used in clinical populations, including participants with PAD, to assess outdoor walking capacity $(8,11)$. The present study formally validated the algorithm for bout detection (8) during outdoor walking sessions, with a 100\% detection rate. 


\section{Accuracy of AG VM raw data and counts for assessing intermittent outdoor walking.}

The AG is the most commonly used accelerometer in PA research and has been extensively studied $(22,40,41)$. However, the literature lacks validation studies for AG in the assessment of short intermittent walking, as proposed in the present study. The existing studies on walking assessment using AG have focused on the identification of walking bouts or the estimation of walking speed during long-duration walking periods $(16,40,42)$, step count identification in intermittent walking (20), or the estimation of MET and energy expenditure at the minute level $(22,41)$. Our study enables researchers to accurately assess intermittent walking using AG. We found a high bout detection rate and an acceptable accuracy for the estimation of walking speed, specifically when using $\mathrm{AG}^{0.033 s, \text { hip }} \mathrm{VM}$ raw data. This finding is consistent with those in previous studies that showed the highest step count accuracy of hip-located over wrist-located AG (20).

Furthermore, with $\mathrm{AG}^{0.033 \mathrm{~s} \text {, hip }} \mathrm{VM}$ raw data, the LMM outperformed the models included in previous studies in estimating walking speed $(16,42)$. Our results show an RMSE of approximately $0.4 \mathrm{~km} / \mathrm{h}$ in the healthy population, a TEE of $0.3[0.27-0.34]$ and a MAPE of $9 \pm$ $6.6 \%$ in the clinical population. Barnett et al. reported that the SEE for walking speed varies between 0.3 and $0.9 \mathrm{~km} / \mathrm{h}$ in free-living $1 \mathrm{~km}$ walking trials assessed using uniaxial AG counts (16).

The present study shows that the AG provides accurate estimations of intermittent outdoor walking parameters in participants with PAD, including the accurate detection of walking and stopping bouts, and an acceptable error in estimating walking speed and distance $(<10 \%)$. The 


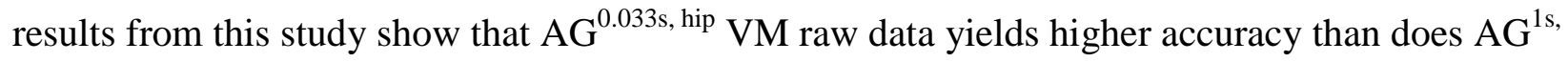
hip VM counts (NF, LFE).

\section{Accuracy of steps for assessing intermittent outdoor walking.}

The SW is considered the most accurate pedometer in estimating steps and has been validated in several contexts, both in healthy and clinical populations $(20,43)$. In addition to the use of steps as a proxy of the walking volume (steps/day), the estimation of speed of ambulation is of interest for a more detailed assessment of walking pattern (21). The speed of ambulation is determined by the combination of cadence (steps/min) and stride length. The use of a pedometer to estimate cadence is possible only with modern, advanced time-stamped pedometers that include a memory function. Furthermore, the way step accumulation is processed by the software according to the sampling epoch has a major impact on which parameter is truly being measured: step accumulation vs. step cadence (44).

There are numerous studies on the step count accuracy of pedometers (17-20), but there are very few studies that addressed their accuracy in the estimation of walking (activity)/stopping duration (45) and cadence $(18,46)$, and to our knowledge, their accuracy in estimating walking speed from cadence is unknown. Our results show that the SW estimations of activity (walking) and inactivity (stopping) durations using proprietary software are largely inaccurate during intermittent walking bouts. This is because the SW software analyzes the data on a minute basis; an active minute is identified if at least one step occurs over the minute considered, and the intensity of ambulation is classified according to the total step count over the same epoch (i.e., one min). In this way, all inactivity durations less than 1 min are neglected by the software, and 
the activity duration is overestimated. Since the total step count (and not cadence) is computed on a minute basis, walking intensity can be inaccurately estimated.

The use of the watershed algorithm significantly improved bout detection with the SW steps, particularly for $\mathrm{SW}^{3 s, \text { ankle }}$ steps, which has the best recording epoch that can be used but allows only 2.5 days of continuous recording. Considering that a recording epoch of $10 \mathrm{~s}$ is the shortest epoch that can be configured for a long recording duration spanning 8 days with this model of $\mathrm{SW}$, our study clearly showed that $\mathrm{SW}^{10 \text { s, ankle }}$ steps had a low accuracy for the identification of intermittent outdoor walking bouts and generally overestimated the walking durations by $27 \%$. The SW steps can better detect walking and stopping bouts of only durations $>2 \mathrm{x}$ the recording epoch, i.e., $6 \mathrm{~s}$ for $\mathrm{SW}^{3 \mathrm{~s} \text {, ankle }}$ and $20 \mathrm{~s}$ for $\mathrm{SW}^{10 \mathrm{~s} \text {, ankle }}$ (data not shown). $\mathrm{AG}^{1 \mathrm{~s} \text {, hip }}$ steps (but not $\mathrm{AG}^{1 \mathrm{~s} \text {, wrist }}$ steps) clearly outperformed $\mathrm{SW}^{3 \mathrm{~s} / 10 \mathrm{~s} \text {, ankle }}$ steps since the steps count was processed over $1 \mathrm{~s}$ epoch. However, the detection rate remained lower than the highest performance obtained using GPS speed, $\mathrm{AG}^{0.033 \mathrm{~s} \text {, hip }} \mathrm{VM}$ raw data and $\mathrm{AG}^{1 \text { s, hip }} \mathrm{VM}$ counts. Since the $\mathrm{AG}$ has been shown to be consistently less accurate than the SW in counting steps $(19,20)$, by capturing either fewer (NF) or more (LFE) steps than the SW, it can be argued that $\mathrm{AG}^{1 \mathrm{~s} \text {, hip }}$ performs better than $\mathrm{SW}^{3 \mathrm{~s} / 10 \mathrm{~s} \text {, ankle }}$ because it does not capture the same (and correct) number of steps. We advocate

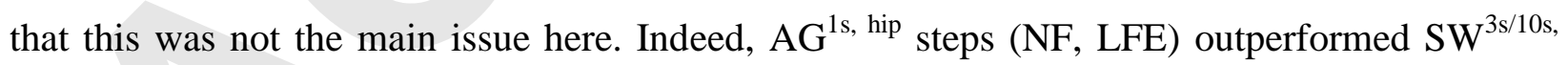
ankle steps in bout detection. Furthermore, when the number of steps captured was compared between $\mathrm{AG}^{1 \mathrm{~s} \text {, hip }}(\mathrm{NF}, \mathrm{LFE})$ and $\mathrm{SW}^{3 \mathrm{~s} \text {, ankle }}$ during each walking bout performed in study 1 , the difference was actually small in the steps count with an MPE and MAPE $\leq 5 \%$ for bout durations >15 s (see Figure, Supplemental Digital Content 4, which illustrates a comparison of steps counting between $\mathrm{AG}^{1 \mathrm{~s}}$, hip steps (NF, LFE) and $\mathrm{SW}^{3 \mathrm{~s},}$ ankle steps, 
http://links.lww.com/MSS/C241). Thus, AG performed better than SW in bout detection mainly due to the shorter recording epoch of the $\mathrm{SW}$, which was particularly true for $\mathrm{SW}^{10 \text { s, ankle }}$. Nevertheless, the RMSE of the estimation of walking speed was very similar between $\mathrm{SW}^{3 \mathrm{~s} \text {, ankle }}$ steps and $\mathrm{AG}^{1 \text { s, hip }}$ steps (NF, LFE), whereas it was higher for $\mathrm{SW}^{10 \text {, ankle }}$ (Table 2).

In the clinical population of PAD participants, all the walking and stopping bouts were $\geq 30 \mathrm{~s}$, and the mean accuracy in bout detection reached $95 \%$ with $\mathrm{SW}^{10 \mathrm{~s} \text {, ankle }}$ steps, with a low and acceptable mean overestimation of the walking duration at the session level by $3 \%$. Conversely, we obtained a higher error (overestimation) in estimating walking speed, with a MAPE of $29 \%$. Again, $\mathrm{AG}^{1 \mathrm{~s} \text {, hip }}$ steps showed higher accuracy in bout detection and lower errors (MAPE) in speed estimation, while the MAPE in the LMM distance estimations were similar between $\mathrm{SW}^{10 s, \text { ankle }}$ steps and $\mathrm{AG}^{1 \mathrm{~s} \text {, hip }}$ steps. Given the limitations of the $\mathrm{SW}$ software in processing the actual activity (walking) duration, the available studies that have proposed a characterization of daily walking pattern in PAD participants from a 7-day period using the $\mathrm{SW}^{10 \mathrm{~s} \text {, ankle }}$ should be reinterpreted accordingly $(47,48)$. Furthermore, during home-based programs in PAD participants, Gardner et al. (7) used the SW "cadence" (which is in fact step accumulation) to estimate MET values over each walking session from a previously determined but unknown individual cadence-speed relationship. Therefore, if the SW software estimates of minute-level activity were used, it cannot be ruled out that the estimation of walking intensity was flawed.

\section{What about the combinations?}

Based on our results, the best combination for assessing intermittent outdoor walking would be $\mathrm{QS}^{1 \mathrm{~s} \text {, hip }}$ speed and $\mathrm{AG}^{0.033 s, \text { hip }} \mathrm{VM}$ raw data, as used in PA studies for other purposes $(26,37)$. 


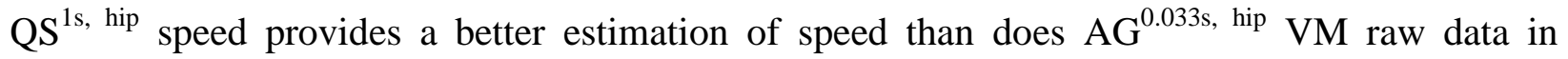

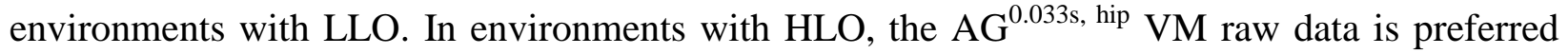
for both walking bout detection and speed estimation. Unless the location of the outdoor walking session is determined in advance and does not change, the use of a GPS is required to have contextual information and determine the level of obstruction of the environment. The environments where the outdoor walking sessions take place can also include a mixture of different levels of obstruction. As we previously observed during outdoor walking sessions of limited duration $(8,11,14,27,32)$, GPS signal dropouts were also a rarity in the present study. However, GPS signal dropouts cannot be totally ruled out, which could preclude the analysis of isolated bouts. The AG can complement or substitute GPS data in such situations. Finally, although energy expenditure was not addressed here, GPS monitors provide higher accuracy in estimating energy expenditure during walking with slopes than do accelerometers (49).

Unless the step count is the expected outcome measure, SW3 should be used with caution when assessing intermittent outdoor walking, specifically if clinical inference is intended (e.g., outdoor walking capacity estimation). SW3 is being replaced by its newest version (SW4), which gives access to the recording of step counting on a second basis and thus can more accurately assess intermittent outdoor walking. This new version is under study. Furthermore, activPAL ${ }^{\text {TM }}$ was not used in the present study but may be an accurate monitor for the assessment of short intermittent outdoor walking in healthy and clinical populations $(13,18)$.

\section{Limitations}

This study has the following main limitations. First, the processing methodology was developed 
and tested for outdoor walking sessions. It cannot be directly applied to detect walking in actual real-world contexts that might include other physical activities. To assess walking activity among other physical activities, additional effort is required to generalize the algorithms to realworld contexts. Second, as previously explained, this study considered outdoor walking only, and our results cannot be extended to indoor contexts. However, the interest in studying indoor walking depends on the final application. For instance, in PAD participants, we previously reported that walking limitations induced by ischemic pain were more likely to occur outdoors (32).

\section{CONCLUSION}

This paper proposed a new method to identify walking and stopping bouts and tested the efficiency of different activity monitors in detecting and estimating intermittent outdoor walking. The results show that QS GPS speed and AG VM raw data can accurately detect walking and stopping bouts as well as estimate walking speed and distance during outdoor walking sessions. These findings have potential importance in clinical applications in which intermittent walking findings are indicative of disease-related outcomes and in which walking is prescribed as an efficient therapeutic strategy. A web platform was developed to help researchers to easily test the proposed methods. A first version of the web platform can be found on the following link: https://mapam.ens-rennes.fr/. 


\section{ACKNOWLEDGMENTS}

Funding. The "Acti-GPS" project was promoted by the University Hospital of Angers and was partially funded by the Genesia Foundation. The "CLASH" project was promoted and partially funded by the University Hospital of Rennes (CORECT 2013). Both the "Acti-GPS" and "CLASH” projects were also partially funded by ENS Rennes. A. Taoum received a postdoctoral

scholarship from the ENS Rennes and the Bretagne region (SAD funding). S. Chaudru received a PhD scholarship from the Bretagne region (ARED funding). P.-Y. de Müllenheim received a PhD scholarship from the ENS Cachan-Antenne de Bretagne (CDSN).

Conflicts of Interest. None. The results of the present study do not constitute endorsement by ACSM. The results of the study are presented clearly, honestly, and without fabrication, falsification, or inappropriate data manipulation. 


\section{REFERENCES}

1. Lee IM, Buchner DM. The importance of walking to public health. Med Sci Sports Exerc. 2008;40(7 SUPPL.1):512-8.

2. Eyler AA, Brownson RC, Bacak SJ, Housemann RA. The epidemiology of walking for physical activity in the United States. Med Sci Sports Exerc. 2003;35(9):1529-36.

3. Kerr J, Sallis JF, Saelens BE, Cain KL, Conway TL, Frank LD, et al. Outdoor physical activity and self rated health in older adults living in two regions of the U.S. Int J Behav Nutr Phys Act. 2012;9:9-12.

4. Saint-Maurice PF, Troiano RP, Bassett DR, Graubard BI, Carlson SA, Shiroma EJ, et al. Association of Daily Step Count and Step Intensity with Mortality among US Adults. JAMA - J Am Med Assoc. 2020;323(12):1151-60.

5. Department of Health \& Human Services. 2018 Physical Activity Guidelines Advisory Committee. Phys Act Guidel Advis Comm Sci Rep [Internet]. 2018;779. Available from: https://health.gov/paguidelines/second-

edition/report/pdf/PAG_Advisory_Committee_Report.pdf

6. Orendurff MS, Schoen JA, Bernatz GC, Segal AD, Klute GK. How humans walk: Bout duration, steps per bout, and rest duration. J Rehabil Res Dev. 2008;45(7):1077-90.

7. Gardner AW, Parker DE, Montgomery PS, Scott KJ, Blevins SM. Efficacy of Quantified Home-Based Exercise and Supervised Exercise in Patients With Intermittent Claudication A Randomized Controlled Trial. Circulation. 2011 Feb 8;123(5):491-8.

8. Le Faucheur A, Abraham P, Jaquinandi V, Bouyé P, Saumet JL, Noury-Desvaux B. Measurement of walking distance and speed in patients with peripheral arterial disease: A novel method using a global positioning system. Circulation. 2008;117(7):897-904. 
9. Barclay R, Webber S, Ripat J, Grant T, Jones CA, Lix LM, et al. Safety and feasibility of an interactive workshop and facilitated outdoor walking group compared to a workshop alone in increasing outdoor walking activity among older adults: A pilot randomized controlled trial. Pilot Feasibility Stud. 2018;4(1):1-16.

10. Dua A, Gologorsky R, Savage D, Rens N, Gandhi N, Brooke B, et al. National assessment of availability, awareness, and utilization of supervised exercise therapy for peripheral artery disease patients with intermittent claudication. J Vasc Surg. 2020 May $1 ; 71(5): 1702-7$.

11. Gernigon M, Le Faucheur A, Noury-Desvaux B, Mahe G, Abraham P. Applicability of global positioning system for the assessment of walking ability in patients with arterial claudication. J Vasc Surg. 2014;60(4):973-981.e1.

12. Clarke CL, Holdsworth RJ, Ryan CG, Granat MH. Free-living physical activity as a novel outcome measure in patients with intermittent claudication. Eur J Vasc Endovasc Surg. $2013 ; 45(2): 162-7$.

13. Granat M, Clarke C, Holdsworth R, Stansfield B, Dall P. Quantifying the cadence of freeliving walking using event-based analysis. Gait Posture. 2015;42(1):85-90.

14. Le Faucheur A, Abraham P, Jaquinandi V, Bouyé P, Saumet JL, Noury-Desvaux B. Study of human outdoor walking with a low-cost GPS and simple spreadsheet analysis. Med Sci Sports Exerc. 2007;39(9):1570-8.

15. Cho GH, Rodríguez DA, Evenson KR. Identifying walking trips using GPS data. Med Sci Sports Exerc. 2011;43(2):365-72.

16. Barnett A, Cerin E, Vandelanotte C, Matsumoto A, Jenkins D. Validity of treadmill- and track-based individual calibration methods for estimating free-living walking speed and 
VO2 using the Actigraph accelerometer. BMC Sports Sci Med Rehabil. 2015;7(1):1-11.

17. Bassett DR, Toth LP, LaMunion SR, Crouter SE. Step Counting: A Review of Measurement Considerations and Health-Related Applications. Sport Med. 2017;47(7):1303-15.

18. Grant PM, Dall PM, Mitchell SL, Granat MH. Activity-monitor accuracy in measuring step number and cadence in community-dwelling older adults. J Aging Phys Act. 2008 Apr 1;16(2):201-14.

19. Toth LP, Park S, Springer CM, Feyerabend MD, Steeves JA, Bassett DR. Video-Recorded Validation of Wearable Step Counters under Free-living Conditions. Med Sci Sports Exerc. 2018;50(6):1315-22.

20. Toth LP, Park S, Pittman WL, Sarisaltik D, Hibbing PR, Morton AL, et al. Effects of Brief Intermittent Walking Bouts on Step Count Accuracy of Wearable Devices. J Meas Phys Behav. 2019;2(1):13-21.

21. Aguiar EJ, Gould ZR, Ducharme SW, Moore CC, McCullough AK, Tudor-Locke C. Cadence-based Classification of Minimally Moderate Intensity during Overground Walking in 21- To 40-Year-Old Adults. J Phys Act Heal. 2019 Dec 1;16(12):1092-7.

22. Matthews CE, Keadle SK, Berrigan D, Staudenmayer J, Saint Maurice P, Troiano RP, et al. Influence of accelerometer calibration approach on MVPA estimates for adults. Med Sci Sports Exerc. 2018;50(11):2285.

23. Sasaki JE, Hickey A, Staudenmayer J, John D, Kent JA, Freedson PS. Performance of Activity Classification Algorithms in Free-living Older Adults. Med Sci Sports Exerc. 2016;48(5):941-50.

24. Ellis K, Kerr J, Godbole S, Staudenmayer J, Lanckriet G. Hip and Wrist Accelerometer 
Algorithms for Free-Living Behavior Classification. Med Sci Sport Exerc. 2016;48(5):933-40.

25. Pavey TG, Gilson ND, Gomersall SR, Clark B, Trost SG. Field evaluation of a random forest activity classifier for wrist-worn accelerometer data. J Sci Med Sport. 2017 Jan 1;20(1):75-80.

26. Hwang LD, Hurvitz PM, Duncan GE. Cross sectional association between spatially measured walking bouts and neighborhood walkability. Int J Environ Res Public Health. 2016;13(4):1-11.

27. Noury-Desvaux B, Abraham P, Mahé G, Sauvaget T, Leftheriotis G, Le Faucheur A. The accuracy of a simple, low-cost GPS data logger/receiver to study outdoor human walking in view of health and clinical studies. PLoS One. 2011;6(9).

28. Serra J. Image analysis and mathematical morphology. Academic Press: New York, USA; 1983.

29. Welk GJ. Principles of Design and Analyses for the Calibration of Accelerometry-Based Activity Monitors. Med Sci Sport Exerc. 2005;37(Supplement):S501-11.

30. Pinheiro JC, Bates DM. Mixed-Effects Models in S and S-PLUS. New York: Springer. 2000.

31. McCrum-Gardner E. Which is the correct statistical test to use? Br J Oral Maxillofac Surg. 2008;46(1):38-41.

32. Chaudru S, Jehannin P, de Müllenheim PY, Klein H, Jaquinandi V, Mahé G, et al. Using wearable monitors to assess daily walking limitations induced by ischemic pain in peripheral artery disease. Scand J Med Sci Sport. 2019;29(11):1813-26.

33. Hopkins WG. Spreadsheets for Analysis of Validity and Reliability. Sportscience. 
$2017 ; 21$.

34. DeShaw KJ, Ellingson L, Bai Y, Lansing J, Perez M, Welk G. Methods for Activity Monitor Validation Studies: An Example With the Fitbit Charge. J Meas Phys Behav. 2018;1(3):130-5.

35. Kang B, Moudon A V., Hurvitz PM, Reichley L, Saelens BE. Walking objectively measured: Classifying accelerometer data with GPS and travel diaries. Med Sci Sports Exerc. 2013;45(7):1419-28.

36. Troped PJ, Oliveira MS, Matthews CE, Cromley EK, Melly SJ, Craig BA. Prediction of activity mode with global positioning system and accelerometer data. Med Sci Sports Exerc. 2008;40(5):972-8.

37. Brondeel R, Pannier B, Chaix B. Using GPS, GIS, and Accelerometer Data to Predict Transportation Modes. Med Sci Sports Exerc. 2015;47(12):2669-75.

38. Hoppe MW, Baumgart C, Polglaze T, Freiwald J. Validity and reliability of GPS and LPS for measuring distances covered and sprint mechanical properties in team sports. PLoS One. 2018;13(2):1-21.

39. Schutz Y, Herren R. Assessment of speed of human locomotion using a differential satellite global positioning system. Med Sci Sports Exerc. 2000;32(3):642-6.

40. Barnett A, Van Den Hoek D, Barnett D, Cerin E. Measuring moderate-intensity walking in older adults using the ActiGraph accelerometer. BMC Geriatr. 2016;16(1):1-9.

41. Crouter SE, Churilla JR, Bassett DR. Estimating energy expenditure using accelerometers. Eur J Appl Physiol. 2006 Dec 21;98(6):601-12.

42. Nichols JF, Morgan CG, Chabot LE, Sallis JF, Calfas KJ. Assessment of Physical Activity with the Computer Science and Applications, Inc., Accelerometer: Laboratory Versus 
Field Validation. Res Q Exerc Sport. 2000;71(1):36-43.

43. Fulk GD, Combs SA, Danks KA, Nirider CD, Raja B, Reisman DS. Accuracy of 2 Activity Monitors in Detecting Steps in People With Stroke and Traumatic Brain Injury. Phys Ther. 2014;94(2):222-9.

44. Dall PM, McCrorie PRW, Granat MH, Stansfield BW. Step accumulation per minute epoch is not the same as cadence for free-living adults. Med Sci Sports Exerc. 2013;45(10):1995-2001.

45. Knarr BA, Roos MA, Reisman DS. Sampling frequency impacts measurement of walking activity after stroke. J Rehabil Res Dev. 2013;50(8):1107-12.

46. Blamey A, Mutrie N. Changing the individual to promote health-enhancing physical activity: The difficulties of producing evidence and translating it into practice. J Sports Sci. 2004 Aug;22(8):741-54.

47. Gardner AW, Montgomery PS, Scott KJ, Afaq A, Blevins SM, City O. Patterns of ambulatory activity in subjects with and without intermittent claudication. J Vasc Surg. 2007 Dec 1;46(6):1208-14.

48. Stansfield B, Clarke C, Dall P, Godwin J, Holdsworth R, Granat M. True cadence and step accumulation are not equivalent: The effect of intermittent claudication on free-living cadence. Gait Posture. 2015 Feb 1;41(2):414-9.

49. de Müllenheim PY, Chaudru S, Emily M, Gernigon M, Mahé G, Bickert S, et al. Using GPS, accelerometry and heart rate to predict outdoor graded walking energy expenditure. J Sci Med Sport. 2018;21(2):166-72. 


\section{FIGURE CAPTIONS}

Figure 1. Example of GPS speed $\left(\mathrm{GS}^{1 \mathrm{~s} \text {, scapula }}\right)^{*}$ for a subject performing the corresponding prescribed walking protocol in the low-level obstruction environment (upper panel) and the assigned walking sequences for the duration-based and distance-based phases in the lower left and right panels, respectively. ${ }^{*} \mathrm{GS}$, GlobalSat DG100 GPS receiver.

Figure 2. Example of the watershed algorithm applied for GPS speed (GS $\left.{ }^{1 \text {, scapula }}\right)^{*}$. The left panel shows $\mathrm{GS}^{1 \mathrm{~s} \text {, scapula }}$ speed data for the duration-based phase of the prescribed walking protocol and the obtained threshold at $1.4 \mathrm{~km} / \mathrm{h}$ (dotted line) representing the local minima of the generated histogram (right panel). *GS, GlobalSat DG100 GPS receiver.

Figure 3. The detection rate versus bout durations for $\mathrm{QS}^{1 \mathrm{~s} \text {, wrist }}$ speed, $\mathrm{AG}^{0.033 \mathrm{~s} \text {, hip }} \mathrm{VM}$ raw data, and $\mathrm{SW}^{3 \text { s, ankle }}$ steps*. The left and right graphs correspond to the environments with low and high levels of obstructions, respectively. The detection rates are displayed with $95 \%$ confidence intervals that were computed using the function binofit in MATLAB ${ }^{2}$ 2018b. *QS, Qstarz BTQ1000XT GPS receiver; AG, ActiGraph wGT3X+ accelerometer; SW, StepWatch3 pedometer. 


\section{SUPPLEMENTAL DIGITAL CONTENT}

Supplemental Digital Content 1. Document that details the design of the first phase of the PWP. pdf

Supplemental Digital Content 2. Document that describes the previous algorithm and its optimization. pdf

Supplemental Digital Content 3. Tables that detail all the results of the study. xlsx

Supplemental Digital Content 4. Figure that illustrates a comparison of steps counting between $\mathrm{AG}^{1 \mathrm{~s}, \text { hip }}$ steps $(\mathrm{NF}, \mathrm{LFE})$ and $\mathrm{SW}^{3 \mathrm{~s} \text {, ankle }}$ steps. pdf 
Figure 1

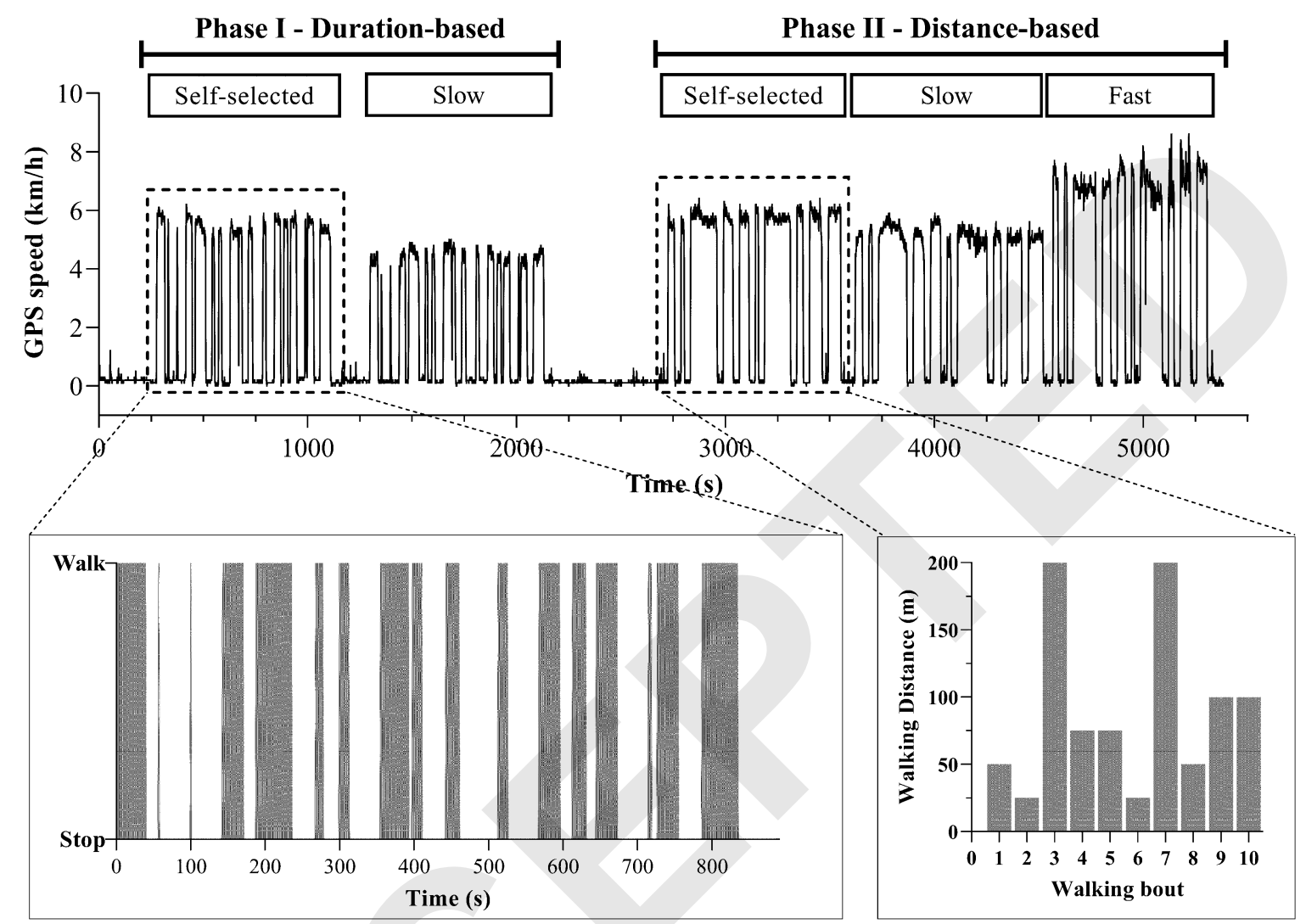


Figure 2
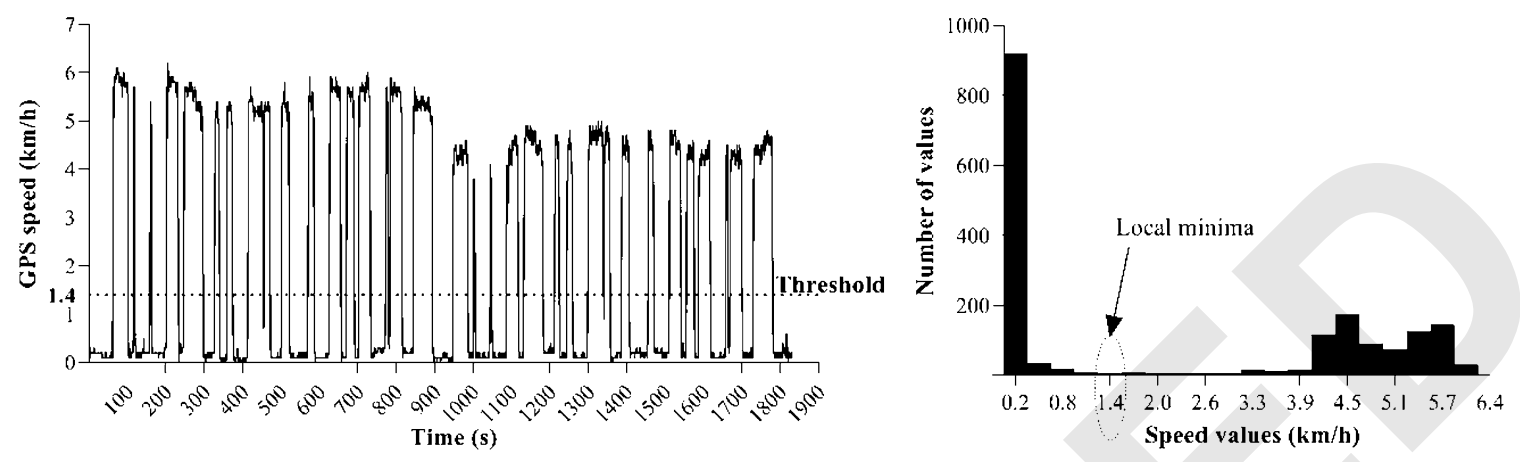
Figure 3
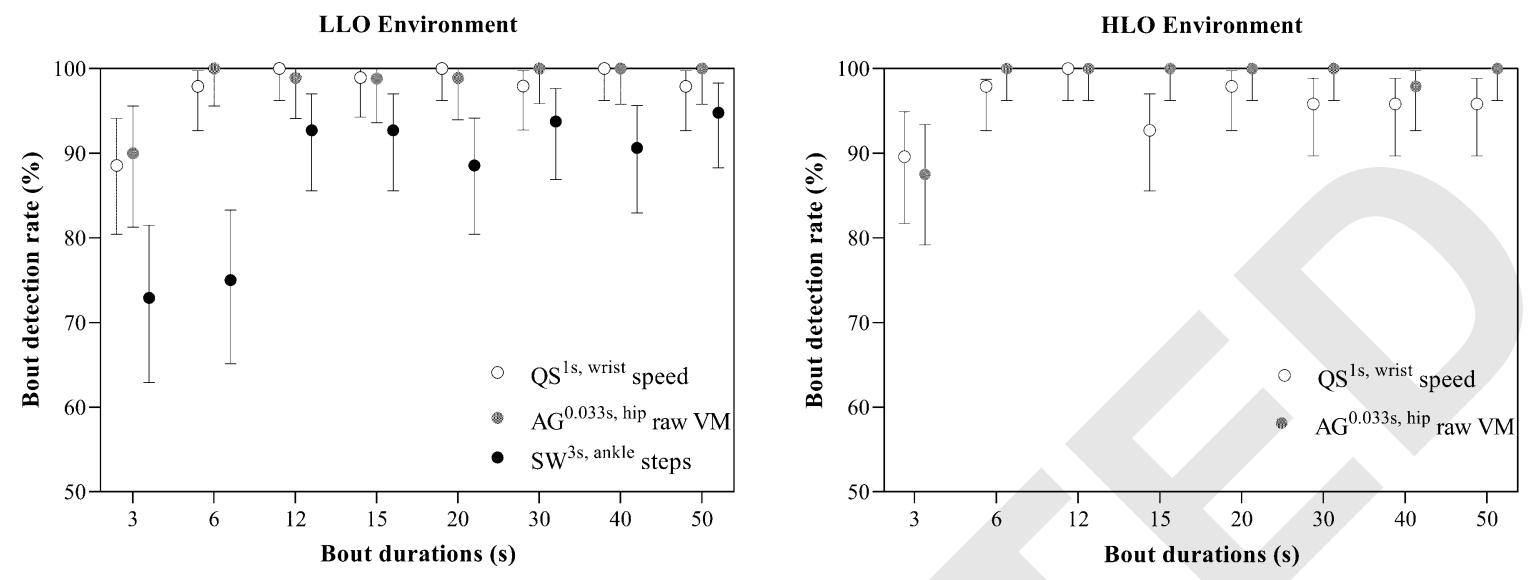
Table 1 - Detection rates obtained with both developed algorithms for walking and stopping bouts according to the different activity monitors and when considering the combination of both environments with low and high levels of obstruction.

\begin{tabular}{|c|c|c|c|c|c|c|}
\hline \multirow[b]{2}{*}{ Monitor } & \multirow[b]{2}{*}{ Parameter $^{\text {a }}$} & \multirow[b]{2}{*}{$\begin{array}{l}\text { Wearing } \\
\text { position }\end{array}$} & \multirow[b]{2}{*}{$\begin{array}{l}\text { Total } \\
\text { bouts }\end{array}$} & \multicolumn{2}{|c|}{ Bout detection rate \% $[95 \% \mathrm{CI}]$} & \multirow[b]{2}{*}{$P$ values } \\
\hline & & & & $\begin{array}{l}\text { Taoum et al. } \\
\text { Watershed } \\
\text { algorithm }\end{array}$ & $\begin{array}{l}\text { Le Faucheur et } \\
\text { al. Enhanced } \\
\text { algorithm }\end{array}$ & \\
\hline GS & Speed (1 s) & Scapula & 1536 & $\begin{array}{l}96.6[95.5- \\
97.4]\end{array}$ & $97.6[96.7-98.3]$ & 0.0025 \\
\hline \multirow{6}{*}{ QS } & \multirow{3}{*}{ Speed (1 s) } & Hip & 1460 & $\begin{array}{c}91.3[89.7- \\
92.7]\end{array}$ & $91.9[90.3-93.2]$ & 0.352 \\
\hline & & Wrist & 1536 & $\begin{array}{c}96.7[95.7- \\
97.5]\end{array}$ & $96.8[95.7-97.6]$ & 0.808 \\
\hline & & Scapula & 1536 & $\begin{array}{c}96.4[95.4- \\
97.3]\end{array}$ & $96.5[95.4-97.4]$ & 0.842 \\
\hline & \multirow{3}{*}{ Speed $(0.1 \mathrm{~s})$} & Hip & 1536 & $\begin{array}{c}90.2[88.6- \\
91.7]\end{array}$ & 88.7 [87.1 - 90.3] & 0.018 \\
\hline & & Wrist & 1536 & $\begin{array}{c}93.4[92.1- \\
94.6]\end{array}$ & 91.1 [89.5 - 92.5] & $<0.001$ \\
\hline & & Scapula & 1536 & $\begin{array}{c}93.9[92.6- \\
95.1]\end{array}$ & $95.4[94.3-96.4]$ & 0.005 \\
\hline \multirow{10}{*}{ AG } & \multirow{2}{*}{$\begin{array}{l}\text { Raw data } \\
(0.033 \mathrm{~s})\end{array}$} & Hip & 1456 & $\begin{array}{c}98.3[97.5- \\
98.9]\end{array}$ & $98.7[98.0-99.2]$ & 0.201 \\
\hline & & Wrist & 1536 & $\begin{array}{c}95.4[94.2- \\
96.4]\end{array}$ & $91.9[90.5-93.2]$ & $<0.001$ \\
\hline & \multirow{2}{*}{ Count NF (1 s) } & Hip & 1456 & $\begin{array}{c}98.2[97.3- \\
98.8]\end{array}$ & 98.3 [97.5 - 98.9] & 0.655 \\
\hline & & Wrist & 1536 & $\begin{array}{c}91.8[90.3- \\
93.1] \\
\end{array}$ & 91.2 [89.7-92.6] & 0.216 \\
\hline & \multirow{2}{*}{ Steps NF (1 s) } & Hip & 1456 & $\begin{array}{l}94.6[93.3- \\
95.7]\end{array}$ & 94.6 [93.3 - 95.7] & 1 \\
\hline & & Wrist & 1536 & $\begin{array}{c}88.3[86.6- \\
89.9]\end{array}$ & $84.4[82.5-86.2]$ & $<0.001$ \\
\hline & \multirow{2}{*}{$\begin{array}{c}\text { Count LFE (1 } \\
\text { s) }\end{array}$} & Hip & 1456 & $\begin{array}{c}97.7 \text { [96.8 - } \\
98.4]\end{array}$ & 99.0 [98.4 - 99.5] & $<0.001$ \\
\hline & & Wrist & 1536 & $\begin{array}{c}92.6[91.2- \\
93.9]\end{array}$ & $92.2[90.7-93.5]$ & 0.307 \\
\hline & \multirow{2}{*}{$\begin{array}{l}\text { Steps LFE }(1 \\
\text { s) }\end{array}$} & Hip & 1456 & $\begin{array}{l}92.9[91.4- \\
94.1]\end{array}$ & $92.9[91.4-94.1]$ & 1 \\
\hline & & Wrist & 1536 & $78.8[76.7-$ & 78.8 [76.7-80.9] & 1 \\
\hline
\end{tabular}




\begin{tabular}{ccccccc}
\hline \multicolumn{1}{c}{ SW } & \multicolumn{6}{c}{$80.9]$} \\
\hline & Steps (3 s) & Ankle & 768 & $\begin{array}{c}87.6[85.1- \\
89.9]\end{array}$ & $80.4[77.4-83.1]$ & $<0.001$ \\
\hline & Steps (10 s) & Ankle & 768 & $\begin{array}{c}21.8[18.9- \\
24.8]\end{array}$ & $30.5[27.2-33.9]$ & $<0.001$ \\
\hline
\end{tabular}

${ }^{\mathrm{a}}$ Recording epoch is indicated for each measured parameter. ${ }^{\mathrm{b}} \mathrm{P}$ values obtained following the McNemar test.

GS, GlobalSat DG100 GPS receiver; QS, Qstarz BT-Q1000XT/-Q1000eX GPS receiver; AG, ActiGraph wGT3X+ accelerometer; SW, StepWatch3 pedometer; NF, normal filter; LFE, low frequency extension filter. 
Table 2- Speed prediction equations developed using Linear Mixed Models along with statistical parameters.

\begin{tabular}{|c|c|c|c|c|c|c|c|}
\hline Model $^{\text {a }}$ & Environment & Equation & $\mathbf{R}^{2}$ & AIC & SEE & RMSE & $P$ values \\
\hline \multirow[t]{3}{*}{ GS $^{1 \mathrm{1s} \text {, scapula }}$ speed } & Combined & $\hat{v}=0.461+0.973 \times v_{m}$ & 0.86 & 158.61 & 0.4 & 0.4 & $<0.001$ \\
\hline & LLO & $\hat{v}_{L L O}=-0.064+1.019 \times v_{m}$ & 0.98 & -274.08 & 0.2 & 0.2 & $<0.001$ \\
\hline & HLO & $\hat{v}_{H L O}=1.051+0.907 x v_{m}$ & 0.82 & 200.96 & 0.3 & 0.4 & $<0.001$ \\
\hline \multirow[t]{3}{*}{$\mathbf{Q S}^{1 \mathrm{~s} \text {, hip }}$ speed } & Combined & $\hat{v}=0.79+0.904 \times v_{m}$ & 0.79 & 540.33 & 0.5 & 0.5 & $<0.001$ \\
\hline & LLO & $\hat{v}_{L L O}=-0.06+1.021 \times v_{m}$ & 0.97 & -175.92 & 0.2 & 0.2 & $<0.001$ \\
\hline & HLO & $\hat{v}_{H L O}=1.822+0.737 \times v_{m}$ & 0.59 & 388.96 & 0.5 & 0.6 & $<0.001$ \\
\hline \multirow[t]{3}{*}{ QS $^{1 \text { s, wrist }}$ speed } & Combined & $\hat{v}=0.299+0.963 \times v_{m}$ & 0.86 & 451.86 & 0.4 & 0.4 & $<0.001$ \\
\hline & LLO & $\hat{v}_{L L O}=-0.155+1.035 \times v_{m}$ & 0.98 & -291.73 & 0.2 & 0.2 & $<0.001$ \\
\hline & HLO & $\hat{v}_{H L O}=1.052+0.831 \times v_{m}$ & 0.63 & 377.30 & 0.5 & 0.5 & $<0.001$ \\
\hline \multirow[t]{3}{*}{ QS ${ }^{1 \text { s,scapula }}$ speed } & Combined & $\hat{v}=0.281+0.989 \times v_{m}$ & 0.84 & 334.65 & 0.4 & 0.4 & $<0.001$ \\
\hline & LLO & $\hat{v}_{L L O}=-0.104+1.026 \times v_{m}$ & 0.99 & -398.01 & 0.1 & 0.1 & $<0.001$ \\
\hline & HLO & $\hat{v}_{H L O}=0.82+0.918 \times v_{m}$ & 0.65 & 340.56 & 0.5 & 0.5 & $<0.001$ \\
\hline $\mathrm{AG}^{0.033 \mathrm{~s}, \text { hip }}$ VM raw data & Combined & $\hat{v}=2.293+9.977 \times r_{m}$ & 0.89 & -139.99 & 0.3 & 0.4 & $<0.001$ \\
\hline $\mathrm{AG}^{0.033 \mathrm{~s} \text {, wrist }} \mathrm{VM}$ raw data & Combined & $\hat{v}=1.727+14.526 \times r_{m}$ & 0.72 & 629.13 & 0.6 & 0.6 & $<0.001$ \\
\hline $\mathrm{AG}^{1 \mathrm{~s}, \text { hip }} \mathrm{VM}$ counts (NF) & Combined & $\hat{v}=1.519+0.044 x c_{m}$ & 0.79 & 293.30 & 0.5 & 0.6 & $<0.001$ \\
\hline $\mathrm{AG}^{1 \mathrm{~s}, \text { wrist }} \mathrm{VM}$ counts (NF) & Combined & $\hat{v}=1.983+0.038 x c_{m}$ & 0.68 & 667.71 & 0.7 & 0.7 & $<0.001$ \\
\hline $\mathrm{AG}^{\text {1s, hip }}$ VM counts (LFE) & Combined & $\hat{v}=1.27+0.046 x c_{m}$ & 0.79 & 294.23 & 0.5 & 0.6 & $<0.001$ \\
\hline $\mathrm{AG}^{1 \mathrm{~s}, \text { wrist }}$ VM counts (LFE) & Combined & $\hat{v}=1.796+0.039 \times c_{m}$ & 0.68 & 660.07 & 0.7 & 0.7 & $<0.001$ \\
\hline $\mathrm{AG}^{1 \mathrm{~s}, \text { hip }}$ steps (NF) & Combined & $\hat{v}=-2.63+0.072 \times \mathrm{cad}_{m}$ & 0.70 & 605.28 & 0.6 & 0.6 & $<0.001$ \\
\hline $\mathrm{AG}^{1 \mathrm{~s} \text {, wrist }}$ steps (NF) & Combined & $\hat{v}=5.96-0.008 \times \mathrm{cad}_{m}$ & 0.01 & 1594.4 & 1.0 & 1.03 & 0.012 \\
\hline $\mathrm{AG}^{1 \mathrm{~s}, \text { hip }}$ steps (LFE) & Combined & $\hat{v}=-4.156+0.084 \times \operatorname{cad}_{m}$ & 0.69 & 531.79 & 0.6 & 0.6 & $<0.001$ \\
\hline $\mathbf{A G}^{1 \mathrm{~s}, \text { wrist }}$ steps (LFE) & Combined & $\hat{v}=3.271+0.019 \times \operatorname{cad}_{m}$ & 0.05 & 1548.27 & 1.0 & 1.0 & $<0.001$ \\
\hline SW $^{3 s, \text { ankle }}$ steps & Combined & $\hat{v}=-4.236+0.087 \times \operatorname{cad}_{m}$ & 0.68 & 348.97 & 0.6 & 0.7 & $<0.001$ \\
\hline $\mathrm{SW}^{10 \mathrm{~s} \text {, ankle }}$ steps & Combined & $\hat{v}=-2.639+0.076 \times \mathrm{cad}_{m}$ & 0.48 & 486.86 & 0.8 & 0.9 & $<0.001$ \\
\hline
\end{tabular}

${ }^{\mathrm{a}}$ The models are presented as the monitors type with the recording epoch and the wearing location as exponents, and the studied parameter.

In equations, $\hat{v},\{v, r, c, c a d\}_{m}$ represent the estimated speed and the studied parameter (GPS speed, $\mathrm{AG}^{0.033 \mathrm{~s}} \mathrm{VM}$ raw data, $\mathrm{AG}^{1 \mathrm{~s}} \mathrm{VM}$ counts, and cadence computed from steps). GS, GlobalSat DG100 GPS receiver; QS, Qstarz BT-Q1000XT GPS receiver; AG, ActiGraph wGT3X+ accelerometer; NF, normal filter; LFE, low frequency extension filter; SW, StepWatch3 pedometer; LLO, low level of obstruction; HLO, high level of obstruction; Combined, low and high level of obstruction environments. 
Table 3-Bout detection rates of walking and stopping in PAD patients for 15 s-filter, and the estimation of speed and distance.

\begin{tabular}{|c|c|c|c|c|}
\hline Parameters & $\begin{array}{c}\text { Detection rate } \\
{\left[\begin{array}{l}95 \% \mathrm{CI}]\end{array}\right.}\end{array}$ & $\begin{array}{c}\text { MAPE (SD) } \\
\text { Speed estimation from LMM }\end{array}$ & $\begin{array}{c}\text { MAPE (SD) } \\
\text { Distance estimation from LMM }\end{array}$ & $\begin{aligned} \text { MAPE }(\text { SD }) \\
\text { Distance }=\text { Estimated speed } * \text { time }\end{aligned}$ \\
\hline $\mathrm{AG}^{1 \mathrm{~s}, \text { hip }} \mathrm{VM}$ counts (NF) & $100[99-100]$ & $9.8(7.3)$ & $12.5(8.5)$ & $10(7.4)$ \\
\hline AG $^{1 \text { s, hip }}$ VM counts (LFE) & $100[99-100]$ & $10.7(8.1)$ & $11.9(7.4)$ & $10.6(8.2)$ \\
\hline $\mathbf{A G}^{0.033 s, \text { hip }} \mathrm{VM}$ raw data & $100[99-100]$ & $9(6.6)$ & $12.5(7.9)$ & $8.4(6.3)$ \\
\hline $\mathbf{A G}^{\text {1s, hip }}$ steps (NF) & 97.8 [95 - 99] & $19.7(10.6)$ & $17.4(9.7)$ & $18.3(10.7)$ \\
\hline SW $^{10 s, \text { ankle }}$ steps & $94.9[92-97]$ & $28.8(11.8)$ & $16.7(10.7)$ & $31.7(11.2)$ \\
\hline
\end{tabular}

GS, GlobalSat DG100 GPS receiver; AG, ActiGraph wGT3X+ accelerometer; NF, normal filter; LFE, low frequency extension filter; SW, StepWatch3 pedometer. 


\section{Supplemental Digital Content 1}

To form the prescribed walking protocols (PWPs), a random sequence alternating between walking and stopping bouts was generated from fixed bout durations of $\{3,6,12,15,20,30$, $40,50\} \mathrm{s}$, with each duration represented 24 times for each class of events (i.e., walk/stop). This resulted into a sequence of 384 ( 8 bouts durations $\times 24 \times 2$ ) walking and stopping bouts that was divided into 10 PWPs lasting each between 10 to $15 \mathrm{~min}$.

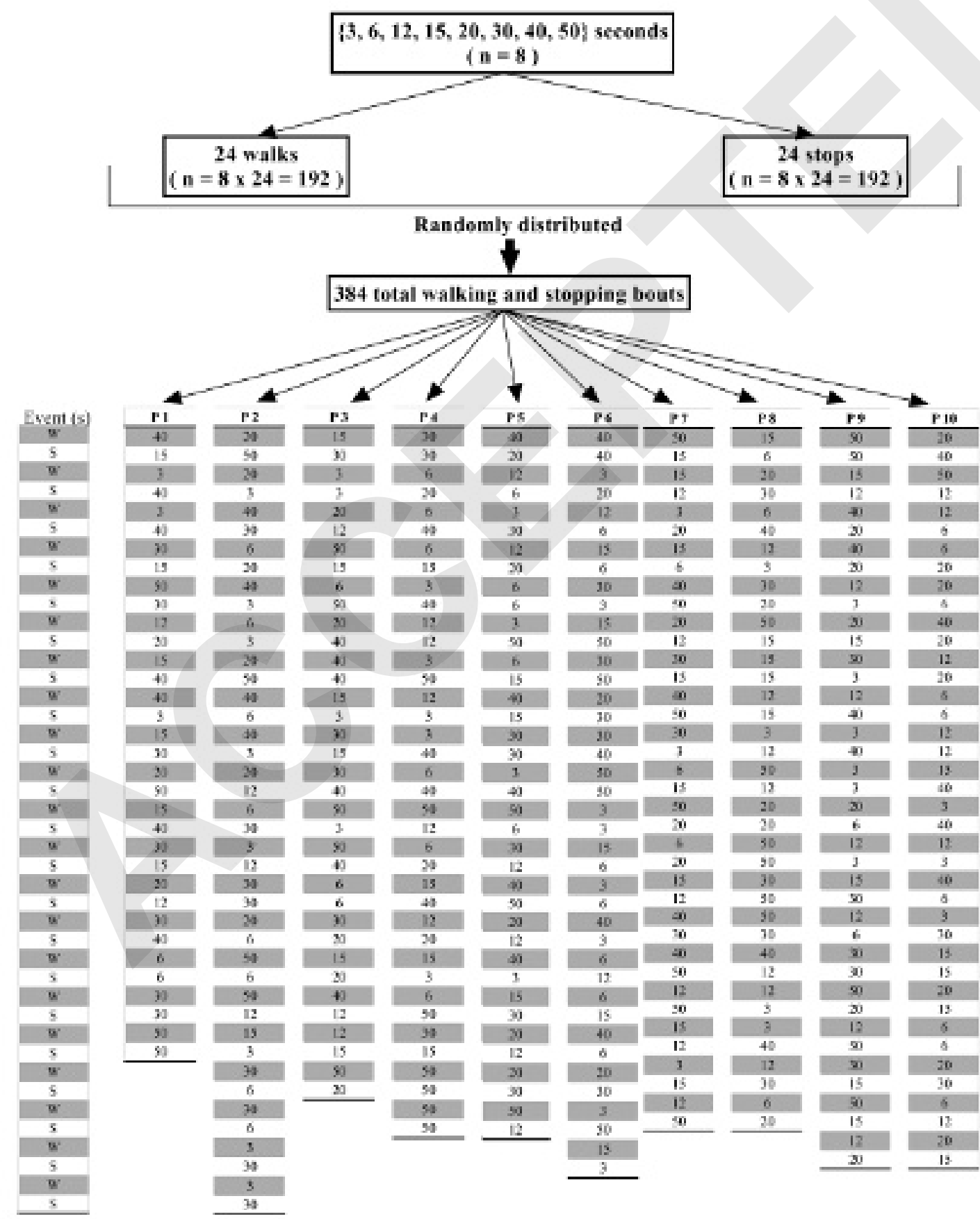

n, number of bouts; W, walking event; S, Stopping event; P, Protocol. 


\section{Supplemental Digital Content 2}

As mentioned in the manuscript, we were interested to enhance our previous method $(1,2)$ by optimizing the detection algorithm and testing it on the different studied parameters from the monitors that were employed in the experiments. The original detection algorithm of this method was the following:

1. The mean of the individual walking speed (IWS) and its standard deviation (SD) were computed from the first walking bout.

2. A low pass filter was applied to remove artifact having values $>2 *$ IWS.

3. A high pass filter was performed to replace all values $<$ IWS $-\mathrm{K}^{*} \mathrm{SD}$ by $0 . \mathrm{K}$ being determined from the coefficient of variation of the IWS $\left(C V_{I W S}\right)$ as follows

$$
K=\left\{\begin{array}{c}
5, \text { if } C V_{I W S}<15 \% \\
2, \text { otherwise }
\end{array} \text {, with } C V_{I W S}=\frac{S D}{I W S} \times 100 .\right.
$$

4. An artifact management was handled to remove short walking and stopping bouts that have duration $<2 *$ recording epoch $(<4$ s for a recording frequency of $0.5 \mathrm{~Hz}$ ).

This method was validated on GPS data in healthy participants $(1,2)$ and then applied for the identification of walking and stopping bouts in participants with PAD during outdoor walking $(3,4)$.

Nevertheless, it highly depends on the IWS and SD computed from the first walking bout, which might create inaccurate identification in case of high variability of walking speed. It also has fixed values of K, which were tested and validated previously for GPS data. Therefore, we proposed to enhance this method to test it on the parameters obtained from all the monitors, as following

1. Computing the mean and SD of parameters for walking bouts: 
a. The watershed algorithm was first applied to preliminary discriminate walking and stopping bouts.

b. The IWS was replaced by the mean of the outputs of the different monitors tested $\left(\mathrm{GS}^{1 \mathrm{~s} \text {, scapula }}\right.$ speed, $\mathrm{QS}^{0.1 \mathrm{~s} \text {, hip/wrist/scapula }}$ speed, $\mathrm{QS}^{1 \mathrm{~s} \text {, hip/wrist/scapula }}$ speed, $\mathrm{AG}^{0.033 \mathrm{~s} \text {, }}$ hip/wrist $\mathrm{VM}$ raw data, $\mathrm{AG}^{1 \mathrm{~s} \text {, hip/wrist }} \mathrm{VM}$ counts and steps, and $\mathrm{SW}^{3 \mathrm{~s} / 10 \text { s, ankle }}$ steps), and was computed along with its SD from all the identified walking bouts for a given subject to take into consideration all the paces performed over a given walking session and thus the measure variability.

2. The low pass filter was applied as in the initial algorithm.

3. The high pass filter was optimized. Instead of having fixed values of $\mathrm{K}$, we have proposed to optimize these values $\left(\mathrm{k}_{1}, \mathrm{k}_{2}\right)$ for each studied parameter using a leave one out cross validation (LOOCV) as follows:

a. From the training set, the CV were computed over the walking bouts of each subject. Then, the $C V_{\text {median }}$ was computed.

b. The optimization of $\left(\mathrm{k}_{1}, \mathrm{k}_{2}\right)$ was performed by minimizing the error rate of the algorithm on the training set using a grid search on $\mathrm{k}_{1}, \mathrm{k}_{2}=\{1,1.5, \ldots, 7\}$.

c. For a test subject, the $C V_{s}$ was computed from the walking data of the subject.

d. The high pass filter was then applied to replace all values $<$ IWS $-\mathrm{K}^{*} \mathrm{SD}$ by 0 , with $K= \begin{cases}k_{1}, & \text { if } C V_{s}<C V_{\text {median }} \\ k_{2}, & \text { otherwise }\end{cases}$

4. The step of artifact management was omitted from this part of analysis due to the presence of very short duration events in the PWPs. 


\section{References}

1. Le Faucheur A, Abraham P, Jaquinandi V, Bouyé P, Saumet JL, Noury-Desvaux B. Study of human outdoor walking with a low-cost GPS and simple spreadsheet analysis. Med Sci Sports Exerc. 2007;39(9):1570-8.

2. Noury-Desvaux B, Abraham P, Mahé G, Sauvaget T, Leftheriotis G, Le Faucheur A. The accuracy of a simple, low-cost GPS data logger/receiver to study outdoor human walking in view of health and clinical studies. PLoS One. 2011;6(9).

3. Le Faucheur A, Abraham P, Jaquinandi V, Bouyé P, Saumet JL, Noury-Desvaux B. Measurement of walking distance and speed in patients with peripheral arterial disease: A novel method using a global positioning system. Circulation. 2008;117(7):897-904.

4. Chaudru S, Jehannin P, de Müllenheim PY, Klein H, Jaquinandi V, Mahé G, et al. Using wearable monitors to assess daily walking limitations induced by ischemic pain in peripheral artery disease. Scand J Med Sci Sport. 2019;29(11):1813-26. 
The following table explains the content of each sheet in this Supplemental Digital Content

\begin{tabular}{|c|c|}
\hline Sheet Name & Content \\
\hline Study1_BoutsDetection & $\begin{array}{l}\text { This sheet presents the bout detection rates }(95 \% \mathrm{CI}) \text { of both algorithms, } \\
\text { Taoum et al. watershed algorithm and Le Faucheur et al. enhanced } \\
\text { algorithm, using all the tested monitors over each duration of walking and } \\
\text { stopping events performed in spontaneous or/and slow paces in environment } \\
\text { with LLO or/and HLO. Thresholds for walking events are also presented as } \\
\text { mean (SD) [min - max] for each monitor using both processing methods. } \\
\text { The CV } \mathrm{V}_{\text {med }}(\%) \text { and }\left\{\mathrm{k}_{1}, \mathrm{k}_{2}\right\} \text { values obtained in Le Faucheur et al. Enhanced } \\
\text { algorithm for each studied parameter are also presented }\end{array}$ \\
\hline Study1_GPSErrors & $\begin{array}{l}\text { This sheet presents the error metrics of measuring walking speed of the GPS } \\
\text { receivers over each distance of walking events performed in spontaneous, } \\
\text { slow, and fast paces in environments with LLO or/and HLO. The error } \\
\text { metrics are the root mean square error (RMSE), the mean percent error } \\
\text { (MPE), the mean absolute percent error (MAPE), the typical error of } \\
\text { estimate (TEE), and the coefficient of variation (CV). MPE and MAPE are } \\
\text { represented with their standard deviation, whereas TEE and CV are } \\
\text { represented with 95\% CI. }\end{array}$ \\
\hline Study1_LMMEquationss & $\begin{array}{l}\text { This sheet presents the LMM predictive equations for speeds and distances } \\
\text { for GPS receivers in environments with LLO or/and HLO, wGT3x } \\
\text { accelerometer data in the combined environments, and SW pedometer data } \\
\text { in LLO environment. }\end{array}$ \\
\hline Study2_BoutsDetection & $\begin{array}{l}\text { This sheet presents the detection rates }(95 \% \mathrm{CI}) \text { of bout identification in the } \\
\text { clinical population of PAD participants for all the monitors, when applying } \\
\text { the filters of } 2,10 \text {, and } 15 \mathrm{~s} \text {. }\end{array}$ \\
\hline Study2_Estimations & $\begin{array}{l}\text { This sheet presents the accuracy of speed and distance estimation from the } \\
\text { LMM predictive equations as well as the estimation of the distance from the } \\
\text { estimated speed (distance = estimated speed } * \text { duration), when applying the } \\
\text { filters of } 2,10 \text {, and } 15 \mathrm{~s} \text {. }\end{array}$ \\
\hline
\end{tabular}

Abbrevations:
\begin{tabular}{|l|l|}
\hline LLO & Low level of obstruction \\
\hline HLO & High level of obstruction \\
\hline NF & Normal filter for wGT3x+ accelerometer \\
\hline LFE & Low filter extension for wGT3x+ accelerometer \\
\hline
\end{tabular}




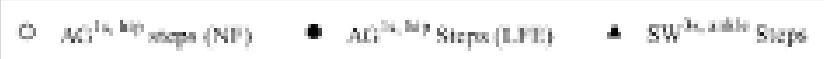
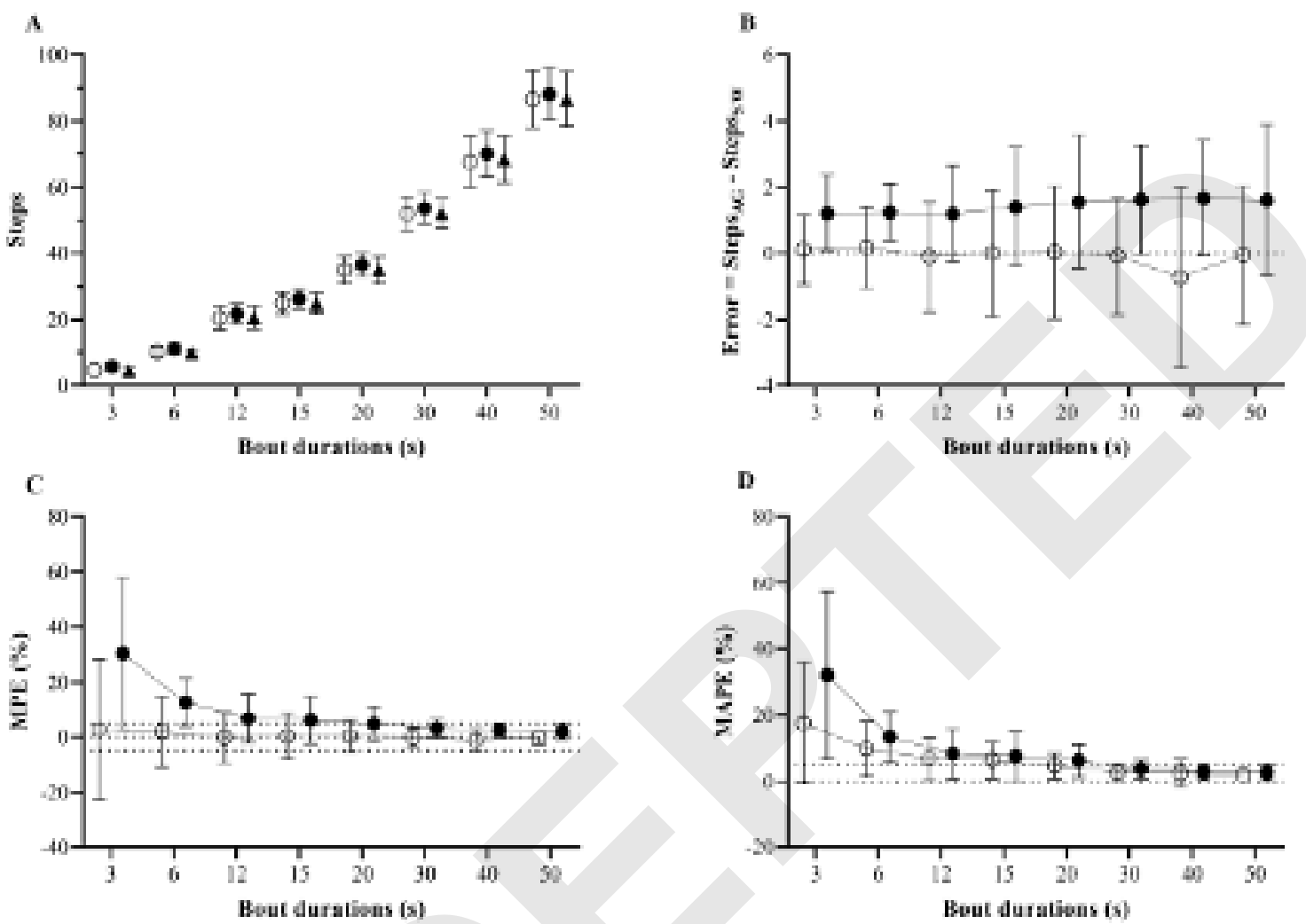

Figure $S$ 1. A comparison of steps counting obtained from $A G^{1 s, \text { hip }}$ steps $(N F, L F E)$ and $S W^{3 s,}$ ankle during the first phase of the prescribed walking protocols in Study 1. Graphs A, B, C, and $D$ represent respectively the steps counting of each studied parameter, the error, MPE (\%), and

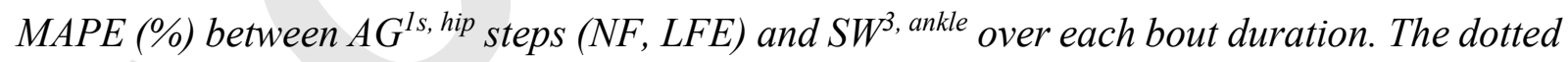
lines in graphs $C$ and $D$ represent the $\pm 5 \%$ error. The graphs show that $A G$ presented a low overestimation of the $S W^{3 s}$ steps when the LFE was enabled, and a low underestimation of $S W^{3 s}$ steps when the LFE is disabled. However, this difference is relatively low with MPE and MAPE $\leq 5 \%$ for bout durations $>15 \mathrm{~s}$. 